vol. $27-n^{\circ} 3 \mid 2011$

Numéro ouvert

\title{
Migrations et emplois à l'île de La Réunion
}

Migration and Employment on Reunion Island

Migración y el empleo en la isla de Reunión

\section{Franck Temporal}

\section{CpenEdition}

Journals

Édition électronique

URL : https://journals.openedition.org/remi/5670

DOI : $10.4000 /$ remi. 5670

ISSN : $1777-5418$

Éditeur

Université de Poitiers

Édition imprimée

Date de publication : 1 décembre 2011

Pagination : 131-164

ISBN : 979-10-90426-02-3

ISSN : 0765-0752

Référence électronique

Franck Temporal, « Migrations et emplois à l'île de La Réunion », Revue européenne des migrations internationales [En ligne], vol. 27 - $n^{\circ} 3$ | 2011, mis en ligne le 01 décembre 2014, consulté le 14 avril 2022. URL : http://journals.openedition.org/remi/5670 ; DOI : https://doi.org/10.4000/remi.5670 


\section{Migrations et emplois à l'île de La Réunion}

\section{Franck TEMPORAL ${ }^{1}$}

Cet article s'interroge sur les relations entre migrations et développement à
l'île de La Réunion à partir d'analyses centrées sur deux dimensions essentielles de la vie des individus, qui sont aussi deux variables clés du développement : l'éducation et l'emploi ${ }^{2}$. Plus précisément, nous cherchons à évaluer dans quelle mesure la migration permet de répondre aux déséquilibres de main-d'œuvre sur le marché du travail selon le niveau de qualification. Après une brève présentation des grandes étapes du développement de l'île, l'objectif est de mesurer l'effet des mouvements migratoires sur la croissance démographique et le volume de la population active ; puis d'évaluer ses conséquences sur la recomposition de la population réunionnaise selon l'âge, l'origine ou encore le niveau de diplôme. Enfin, l'objet de la dernière partie est d'apprécier l'incidence de la migration sur l'accès à l'emploi et le type d'emploi occupé par les individus à partir d'une comparaison des populations migrantes et non migrantes.

Cette analyse quantitative repose essentiellement sur l'exploitation des données des recensements de la population jusqu'en 1999, dernier recensement mené à l'exhaustif' . Plus précisément nous comparons plusieurs sous-populations :

- Les natifs de La Réunion de retour de migration

- Les natifs de La Réunion non migrants

- Les immigrants non natifs à La Réunion parmi lesquels nous distinguons : les individus nés en métropole ; les individus nés à l'étranger; les individus nés dans un autre Dom-Tom - Les émigrants réunionnais installés en métropole

Les critères du lieu de naissance et du lieu de résidence antérieure permettent de déterminer les populations migrantes. Nous qualifions d'immigrants, les personnes nées hors de La Réunion, y résidant. Nous qualifions d'émigrants, les natifs de La Réunion

1 Contractuel INED, 133 boulevard Davout 75020 Paris ; franck.temporal@ined.fr

2 Cet article fait la synthèse d'une analyse plus large menée dans le cadre d'un doctorat de démographie soutenu en juin 2008 à l'université Paris Descartes, disponible auprès de l'auteur.

3 Les premières analyses menées à partir des fichiers détails du recensement de 2006 indiquent des résultats proches de ceux observés à partir des recensements précédents, les grandes tendances présentées sont respectées. 
résidant hors de leur département. Le croisement des variables du lieu de résidence antérieure (au recensement précédent) et du lieu de naissance nous permet d'isoler, même de façon imparfaite 4 , les natifs de La Réunion de retour de migration, c'est-à-dire ceux qui, après avoir connu une migration, sont revenus s'installer sur l'île entre deux recensements.

\section{LES GRANDES ÉTAPES DU DÉVELOPPEMENT DEPUIS LA DÉPARTEMENTALISATION}

Pour comprendre la situation actuelle de ce département français de l'Océan Indien, il importe de présenter brièvement les grandes étapes du développement sanitaire, économique et social de ces dernières décennies. La Réunion a en effet connu des transformations rapides et profondes de sa société dont les évolutions sont sans aucune mesure avec celles observées au niveau national sur la même période. Le développement de La Réunion est indissociable de la politique de départementalisation, qualifiée de rattrapage, dont l'objectif principal visait à combler les retards sur le modèle des départements de métropole. En un demi-siècle (1946-1999), l'île a connu de profondes mutations et des transitions multiples (politique, épidémiologique, démographique, familiale, économique et sociale) dont la caractéristique commune est leur simultanéité et leur rapidités.

\section{Une révolution sanitaire déclenche la transition démographique}

Le premier objectif de l'État français a été d'améliorer l'état sanitaire et les conditions d'hygiène en luttant contre les parasites, en lançant des campagnes d'alimentation et de vaccination et en développant un système de soins performant. L'amélioration des conditions de vie est passée également par la mise en place et le développement des infrastructures routières, sanitaires et éducatives (Lopez, 1996). On assiste à la construction d'hôpitaux, de centres de PMI, de dispensaires, d'écoles. Les actions menées dans le domaine du logement, notamment en matière de lutte contre l'habitat insalubre, vont permettre d'améliorer les conditions de vie. Les grands travaux d'équipement en électricité, de raccordement aux réseaux d'eau potable et d'évacuation des eaux usées font progresser le confort des logements. Grâce à l'effort financier accordé par l'État à partir des années 1950-1960, peu à peu la situation sanitaire et alimentaire de l'île s'améliore pour atteindre un niveau de développement comparable aux départements métropolitains à la fin des années 1990. Dans le même temps, La Réunion va connaître une transition épidémiologique très rapide ${ }^{6}$. Ces progrès sanitaires ont eu pour conséquence une réduction rapide de la mortalité suivie de celle de la natalité.

4 La source « recensement » sous-estime le nombre total de retour car à la différence des enquêtes spécifiques elle ne tient compte que des retours enregistrés entre deux opérations de recensements et ne permet pas d'identifier les aller-retour qui ont eu lieu entre les deux dates de collecte.

5 Ces bouleversements se sont accompagnés de nombreux changements (mise en place d'un système éducatif moderne, développement de l'offre de soins, amélioration du confort des logements, etc.) qui ont eu un effet positif sur le développement.

6 Alors que le paludisme représentait la première cause de décès en 1950, La Réunion se trouve aujourd'hui protégée de la majorité des maladies infectieuses ; ce sont désormais les maladies de civilisation (diabète, obésité, maladies cardio-vasculaires, tumeurs et accidents) qui prédominent (Catteau, 2003). 
La transition démographique de La Réunion, toujours en voie d'achèvement, a été courte en durée et haute en accroissement (Festy et Hamon, 1983). Au moment de la départementalisation (1946), le taux de mortalité chute tandis que la natalité connaît une hausse spectaculaire en raison de l'amélioration des conditions sanitaires (Figure 1). La Réunion connaît alors un accroissement démographique soutenu et la croissance naturelle supplante, pour la première fois, l'effet de l'immigration. La deuxième phase (1952-1967) représente le cœur de la transition démographique réunionnaise. C'est au cours de cette période que va se produire «l'explosion démographique ». Le taux de mortalité poursuit sa tendance à la baisse, tandis que la natalité diminue peu. L'accroissement naturel dépasse alors $30 \%$, et, en l'espace de quinze ans, la population réunionnaise augmentera de plus de moitié. Depuis les années 1970, la fin de transition s'attarde en raison du nombre élevé des naissances qui s'explique par l'importance de la population en âge de procréer, malgré un vieillissement démographique émergent (Tableau 1). Entre 1967 et 1982, la croissance démographique va ralentir sa progression, sous le double effet du ralentissement de l'accroissement naturel et de l'émigration massive des jeunes réunionnais vers la métropole. Depuis les années 1980, les tendances se poursuivent, mais à un rythme moindre. La mortalité diminue peu et la natalité se maintient en raison d'un niveau de fécondité relativement haut, notamment avant vingt-cinq ans (Breton et Temporal, 2010). Au total, la population de La Réunion a presque été multipliée par trois depuis 1946.

Figure 1 : Les grandes phases de la transition démographique à La Réunion

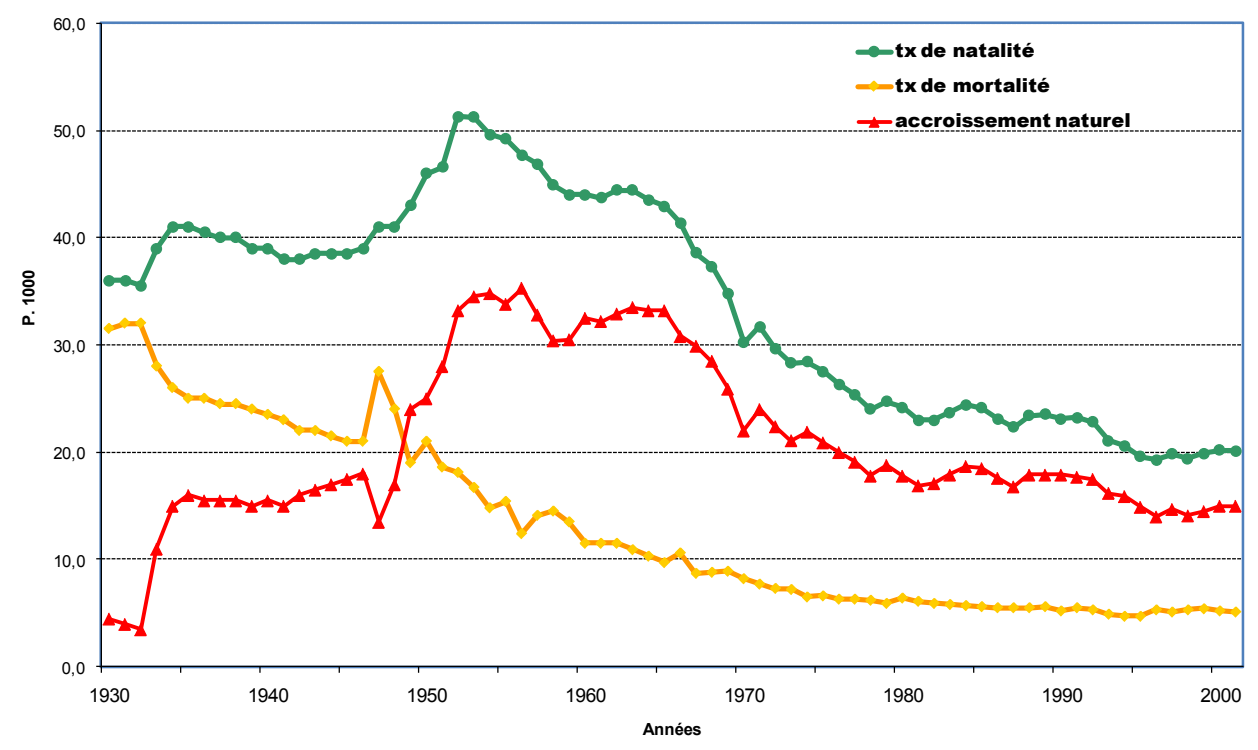

Source : INSEE, Recensements de la population et État Civil. 
Tableau 1 : Principaux mouvements naturels de 1946 à 1999

\begin{tabular}{|c|c|c|c|c|c|c|c|c|}
\hline & 1946 & 1954 & 1961 & 1967 & 1974 & 1982 & 1990 & 1999 \\
\hline $\begin{array}{l}\text { Population au RP, } \\
\text { dont : }\end{array}$ & 241708 & 274370 & 349282 & 416525 & 476675 & 515814 & 597823 & 706300 \\
\hline $\begin{array}{l}- \text { les moins de } \\
20 \text { ans (\%) }\end{array}$ & 49 & 52,1 & 54,3 & 56,4 & 53,6 & 49,1 & 39,9 & 36,2 \\
\hline - $20-59$ ans $(\%)$ & 46,0 & 42,6 & 40,5 & 38,4 & 40,3 & 44,0 & 51,5 & 53,8 \\
\hline $\begin{array}{l}- \text { les plus de } \\
60 \text { ans }(\%) \\
\end{array}$ & 5,0 & 5,3 & 5,2 & 5,2 & 6,1 & 6,9 & 8,6 & 10,0 \\
\hline $\begin{array}{l}\text { Taux d'accrois- } \\
\text { sement annuel (\%) }\end{array}$ & 0,4 & 2,5 & 3,5 & 3,0 & 2,1 & 0,9 & 1,9 & 2,0 \\
\hline $\begin{array}{l}\text { Taux de mortalité } \\
(\%)\end{array}$ & 21,0 & 14,8 & 11,5 & 8,7 & 6,5 & 5,9 & 5,2 & 5,4 \\
\hline $\begin{array}{l}\text { Taux de natalité } \\
(\%)\end{array}$ & 39,0 & 49,6 & 43,7 & 38,6 & 28,4 & 23,0 & 23,1 & 19,9 \\
\hline $\begin{array}{l}\text { Accroissement } \\
\text { naturel }(\%)\end{array}$ & 18,0 & 34,8 & 32,2 & 29,9 & 21,9 & 17,1 & 17,9 & 14,5 \\
\hline $\begin{array}{l}\text { Indice Synthétique } \\
\text { de Fécondité } \\
\text { (nombre moyen } \\
\text { d'enfants par femme) }\end{array}$ & 5,6 & 6,9 & 6,4 & 6,1 & 3,9 & 2,9 & 2,6 & 2,3 \\
\hline
\end{tabular}

Source : INSEE, Recensements de la population et État Civil.

\section{La transformation de l'économie et la montée du chômage}

Dans le même temps, la transformation de l'économie a été très marquée. Au moment de la départementalisation, la société réunionnaise était rurale, l'agriculture et notamment la plantation - fournissait l'essentiel des emplois ${ }^{7}$. La part des emplois agricoles a fortement chuté depuis. En 1999, l'agriculture représente seulement 5,5\% des emplois, le secteur secondaire $14 \%$ et les services plus de $80 \%$. Au cours de cette modernisation de l'économie, la croissance a été forte : entre 1974 et 1999, l'Insee évalue la croissance économique à $5 \%$ par an en moyenne (AFD-INSEE-IEDOM, 2004). Pour autant, c'est le niveau élevé du chômage et non le dynamisme économique qui constitue la spécificité de l'île. Le rythme de progression du chômage a été plus soutenu que celui des emplois créés ${ }^{8}$. L'augmentation du nombre de chômeurs a été une réalité inéluctable pendant plus de trente ans, de $13 \%$ en 1967, le taux de chômage a augmenté jusqu'à

$767 \%$ de la population active occupée.

8 Entre les recensements de 1967 et 1999 , la population active a augmenté de $175 \%$, la population active occupée de $84 \%$ et le nombre de chômeurs de plus de $770 \%$. 
$42 \%$ de la population active en $1999^{9}$. Cette forte hausse s'explique par l'évolution du marché de l'emploi, la hausse de la population active, mais aussi par la représentation du chômage. En effet, pendant l'économie de plantation les périodes de sous-emploi ou d'inactivité étaient nombreuses ${ }^{10}$, mais elles étaient alors moins considérées comme du « chômage » (Parain, 1996). Néanmoins, quelle que soit la définition utilisée, le niveau très élevé du chômage en fait la principale préoccupation économique et sociale de l'île.

Comme le note l'Insee, l'Iedom et l'Afd dans une publication commune, trois principaux facteurs expliquent la montée du chômage au cours de cette période. Le premier est la croissance démographique et notamment l'augmentation de la population en âge de travailler; le second est la hausse des taux d'activité en raison de l'entrée des femmes sur le marché du travail ; enfin, la hausse de la productivité a entraîné un écart important entre le rythme de la croissance économique et celui de la création d'emplois (Insee-Iedom-Afd, 2004).

Un décalage important existe donc entre l'offre et la demande de travail à La Réunion, que la croissance économique - pourtant forte - n'a pas permis de combler. Si la création d'emplois a été soutenue, elle est restée trop faible pour absorber les flux de nouveaux actifs arrivant chaque année sur le marché du travail et le stock de chômeurs. On se trouve ainsi dans un contexte particulier, voire paradoxal, où la croissance économique forte s'est accompagnée de déséquilibres structurels (déficit commercial) et humains (chômage, pauvreté) importants. Ainsi, selon les indicateurs macro du développement tels que le « PIB/habitant» ou encore de l'« Indice de Développement Humain (IDH) », l'île appartient résolument à l'ensemble des pays développés. Elle affiche un niveau de développement nettement supérieur aux pays de l'Océan Indien constituant son environnement proche, même si elle figure parmi les régions les moins développées de France et d'Europe. Les instances européennes et nationales lui reconnaissent des handicaps structurels spécifiques ${ }^{11}$, comme les autres régions ultrapériphériques (RUP) ${ }^{12}$, justifiant le niveau élevé des transferts publics qui constituent le principal moteur de l'économie, permettent de réduire les déséquilibres et de maintenir les niveaux de vie. Cependant, ces indicateurs synthétiques de mesure du développement au niveau macro sont peu adaptés au contexte réunionnais, car ils ne permettent pas d'appréhender les déséquilibres au niveau micro, et, notamment, le niveau élevé du chômage et la persistance d'inégalités marquées entre individus et groupes sociaux.

$9 \mathrm{Au}$ sens du recensement de la population, est considérée comme chômeur, toute personne qui se déclare spontanément comme tel, sauf si elle a déclaré par ailleurs ne pas rechercher de travail. Depuis 1982, le Bureau International du Travail (BIT) a défini des critères plus restrictifs du chômage : est considérée comme chômeur toute personne âgée de quinze ans ou plus qui n'a pas travaillé pendant la semaine de référence, est disponible pour occuper un emploi dans les quinze jours et a recherché activement un emploi au cours du mois précédent. En 1999, le taux de chômage au sens du BIT était de 37,7 \%. La notion de chômage au sens du recensement est donc plus large que celle utilisée dans les enquêtes emploi.

10 En raison de la saisonnalité de l'économie de plantation.

11 Notamment les handicaps liés à l'insularité et à l'éloignement : isolement, éloignement, étroitesse du marché intérieur, etc.

12 L'Europe compte sept régions ultrapériphériques (RUP) : les quatre DOM français (Guadeloupe, Martinique, Guyane, Réunion), la communauté autonome espagnole des îles Canaries et les deux régions autonomes portugaises des Açores et de Madère. 


\section{L’approche individuelle du développement : des inégalités encore marquées}

Si le niveau de formation a considérablement progressé à La Réunion, plusieurs études indiquent que les inégalités en matière d'éducation restent fortes (Cheung Chin Tun, 2000 ; Payet, 2000). Le taux d'analphabétisme des personnes de plus de quinze ans se maintient à un niveau élevé : $24 \%$ en 1988 et $23 \%$ en 1996. Par ailleurs, en 2002, l'évaluation des Journées d'appels et de préparation à la Défense indique que $22,5 \%$ des jeunes ont été repérés en " difficultés de lecture » dont 10,3\% en " grandes difficultés ». La problématique de l'illettrisme chez ces jeunes ayant été scolarisés renvoie plus généralement aux problèmes d'échec scolaire : en 2002, $15 \%$ des jeunes sortent du système scolaire sans qualification ${ }^{13}$, le lien étant marqué entre la situation d'activité des parents et les performances scolaires des jeunes générations. Les inégalités sociales produisent des inégalités scolaires qui vont avoir à leur tour une influence sur les possibilités d'insertion professionnelle des individus.

Le chômage touche fortement les générations les plus jeunes qui, bien que plus diplômées que leurs aînées, souffrent de leur arrivée récente sur un marché de l'emploi de plus en plus saturé. À ces inégalités selon l'âge s'ajoute une différence selon le sexe : à âge égal, les femmes sont plus confrontées au chômage que les hommes ${ }^{14}$. Dans une société où le nombre des inactifs ne se déclarant pas au chômage est élevé, la lecture des taux d'emploi permet de souligner l'ampleur du non-emploi. En 1999, seulement 43,5\% des hommes et $30,7 \%$ des femmes âgés de quinze à soixante-quatre ans exercent une activité. Les jeunes générations éprouvent les plus grandes difficultés d'accès à l'emploi : seulement un quart des hommes âgés de vingt à vingt-quatre ans et un peu plus de la moitié des vingt-cinq à vingt-neuf ans occupent un emploi. La situation est plus difficile encore pour les femmes ${ }^{15}$. Entre vingt-sept et trente-sept ans, les écarts de taux d'emploi entre la métropole et La Réunion sont maxima, ils dépassent les 30 points de différence. À ces différentiels déjà importants s'ajoutent des écarts sensibles selon le niveau de formation des individus ${ }^{16}$. En 1999, le taux de chômage des bacheliers atteint 24 \%, celui des diplômés du premier cycle, $12,8 \%$, celui des diplômés du deuxième ou troisième cycle, seulement 5,9\%, alors que celui des non diplômés s'établit à 55,3\%. L'écart entre les titulaires de diplômes les plus élevés et ceux qui n'ont pas de qualification est très prononcé (49,4 points d'écart en 1999).

13 Soit une proportion deux fois plus élevée que la moyenne des départements de métropole (Académie de La Réunion, 2004).

14 À vingt ans, $71 \%$ des hommes et $75 \%$ des femmes se déclarent au chômage au sens du recensement ; à vingt-cinq ans, $48 \%$ des hommes et $54 \%$ des femmes sont concernés ; à trente ans, ces taux s'établissent respectivement à $42 \%$ et $51 \%$. ; enfin, à trente-cinq ans, $36 \%$ des hommes et 46 \% des femmes sont chômeurs. À cinquante-neuf ans, le chômage touche encore un quart des actifs.

15 Seulement $21 \%$ d'entre elles ont un emploi dans le groupe vingt/vingt-quatre ans et $37 \%$ à vingt-cinq/vingt-neuf ans.

16 Jusqu'au début des années 1980, le chômage touchait presque exclusivement les personnes les moins formées. On assiste depuis à une hausse du chômage des diplômés. Ces derniers représentent cependant moins de $9 \%$ des chômeurs en 1999, contre $67 \%$ pour les personnes pas ou peu diplômées (sans diplôme et titulaires du Certificat d'études primaires). 
Les jeunes diplômés ne sont donc plus assurés d'accéder directement à l'emploi, mais le diplôme reste la meilleure des protections face à la progression du chômage.

Conséquence du faible accès à l'emploi, le nombre de bénéficiaires de minima sociaux est élevé (Tableau 2) : $19 \%$ de la population totale et $30 \%$ des individus de vingt ans ou plus en $2003^{17}$. Plusieurs de ces minima sont nettement surreprésentés dans le département. C'est le cas du Revenu minimum d'insertion (RMI) qui concerne 9,3\% de la population totale (moins de $2 \%$ en métropole) et touche près de $25 \%$ des individus si l'on tient compte des ayants droit. L'Allocation de solidarité spécifique (ASS) dont bénéficient les chômeurs de longue durée ${ }^{18}$ et l'Allocation supplémentaire vieillesse (AVS) concerne aussi beaucoup plus fréquemment les Réunionnais que les métropolitains ${ }^{19}$.

Tableau 2 : Bénéficiaires des minima sociaux au 31 décembre

\begin{tabular}{|l|c|c|c|c|}
\hline & $\mathbf{1 9 9 0}$ & $\mathbf{2 0 0 1}$ & $\mathbf{2 0 0 2}$ & $\mathbf{2 0 0 3}$ \\
\hline Revenu minimum d'insertion (RMI) & 50265 & 63483 & 67915 & 70851 \\
\hline Revenu de solidarité (RSO) & $/ / /$ & 4633 & 5398 & 5447 \\
\hline Allocation de parent isolé (API) & 7555 & 6466 & 7206 & 7940 \\
\hline Allocation adulte handicapé (AAH) & 8585 & 10528 & 10536 & 10580 \\
\hline Allocation de solidarité spécifique (ASS) & 1824 & 13109 & 13062 & 13073 \\
\hline Allocation d'insertion (AI) & $/ / /$ & 162 & 207 & 188 \\
\hline Allocation supplémentaire vieillesse* & 28699 & 33832 & 33106 & 34155 \\
\hline \multicolumn{1}{|c|}{ dont Allocation spéciale vieillesse } & $/ / /$ & 3384 & 3550 & 3592 \\
\hline Allocation veuvage & 391 & 294 & 274 & 215 \\
\hline Minimum invalidité** & 2683 & 1975 & 2031 & 2169 \\
\hline \multicolumn{1}{|c|}{ Total des bénéficiaires } & $\mathbf{1 0 0} \mathbf{0 0 2}$ & $\mathbf{1 3 4} \mathbf{4 8 2}$ & $\mathbf{1 3 9} \mathbf{7 3 5}$ & $\mathbf{1 4 4} \mathbf{6 1 8}$ \\
\hline $\begin{array}{l}\text { Part des bénéficiaires de minima sur la population } \\
\text { de 25 ans et + (en \%) }\end{array}$ & 33,4 & 32,8 & 33,5 & 34,1 \\
\hline $\begin{array}{l}\text { Part des bénéficiaires de minima sur la population } \\
\text { de 20 ans et + (en \%) }\end{array}$ & 28 & 28,8 & 29,3 & 29,7 \\
\hline
\end{tabular}

(*) Régime général, régime agricole et allocation spéciale vieillesse (sauf pour 1990).

(**) Régime général et agricole.

Source : CAF de La Réunion, CGSS, ASSEDIC, INSEE.

Globalement, la politique de rattrapage social menée à La Réunion au cours des vingt-cinq dernières années a permis de réduire les inégalités de revenus. Cependant, la tendance indique un ralentissement des progrès à la fin des années 1990 et les indices de

17 Cette part est nettement plus élevée qu'en France métropolitaine (5\% de la population totale).

18 La part des allocataires de l'ASS dans la population active est de 4,4 \% à La Réunion $(1,4 \%$ en métropole), ce qui en fait le département français où cette proportion est la plus élevée (Temporal, 2006).

19 À La Réunion, $62 \%$ des individus âgés de soixante-cinq ans ou plus bénéficient du minimum vieillesse, cette part n'est que de $7 \%$ en métropole. 
pauvreté monétaire stagnent, malgré une croissance économique soutenue et une augmentation sensible des transferts publics en direction des plus démunis ${ }^{20}$.

Ce contexte de concurrence forte sur un marché du travail saturé se traduit donc par une mise à l'écart des personnes les plus défavorisées (pas ou peu diplômés, chômeurs et inactifs de longue durée), mais aussi par des phénomènes de nivellement et de déclassement qui touchent les diplômés. Certains individus acceptent, faute de mieux, des emplois précaires ou des emplois d'un niveau de qualification inférieur à la valeur de leur diplôme (Parain, 1999). Cette dévaluation des titres scolaires ne concerne pas seulement les individus les moins diplômés. Le chômage et les inégalités sociales sont des déterminants essentiels de la pauvreté et de l'exclusion, ils constituent deux défis majeurs du développement pour La Réunion (Temporal, 2006). L'approche individuelle du développement indique donc que les progrès observés au niveau macro n'ont pas profité également à toutes les catégories de la population et que les inégalités en matière d'éducation et d'accès à l'emploi restent importantes.

\section{MIGRATIONS ET CROISSANCE DÉMOGRAPHIQUE (1946-1999)}

Dans ce contexte de concurrence entre individus et de croissance de la population en âge de travailler, quelle place et quels effets ont les migrations ?

\section{La politique et la gestion institutionnelle de la migration}

À La Réunion, comme dans nombre de sociétés insulaires confrontées à une forte croissance démographique et au problème du sous-emploi, la migration a toujours constitué une dimension essentielle de la politique de développement en tant que variable d'ajustement des déséquilibres de main-d'œuvre (excédent ou pénurie). La logique d'implantation, tout d'abord, avec les premiers arrivants aux origines du peuplement chargés de la mise en valeur de l'île. L'immigration forcée, puis libre ensuite, devant répondre aux besoins importants en main-d'œuvre de l'économie de plantation. L'immigration encore, au moment de la politique de départementalisation, motivée par la pénurie de travailleurs qualifiés, qui s'est traduite par la mise en place de compléments de rémunération des fonctionnaires de l'État travaillant dans les $\mathrm{DOM}^{21}$. L'émigration des natifs enfin, avec

20 La part des ménages vivant en dessous du seuil de pauvreté est stable selon les enquêtes budget des familles de l'Insee : $10 \%$ en 1995 et en 2001. L'écart entre les ressources des ménages les plus aisés et des plus démunis reste important à La Réunion, il est supérieur à celui observé au plan national.

21 Des compléments de rémunération des salariés de la fonction publique d'État spécifiques aux DOM ont été instaurés dans les années 1950 pour répondre aux importants besoins de maind'œuvre qualifiée - que l'île ne pouvait fournir en raison du faible niveau de formation de la population - dans les secteurs de l'éducation, de la santé, de l'action sociale et des principales administrations ; à La Réunion, le taux de majoration de traitement des fonctionnaires de l'État atteint $53 \%$ (rapport parlementaire du député Brard, 2007). 
les expériences de la $\mathrm{SAKAY}^{22}$, du BUMIDOM ${ }^{23}$, du $\mathrm{CNARM}^{24}$, de l'ANT $^{25}$, jusqu'à la politique de mobilité actuelle ${ }^{26}$ dont les objectifs ont toujours été de lutter contre le sousemploi d'une population en forte croissance.

\section{L'évolution de la migration à travers les soldes migratoires intercensitaires}

L'évolution du solde migratoire intercensitaire (Tableau 3) permet de comprendre comment cette gestion institutionnelle des migrations s'est vérifiée dans les faits. Le solde migratoire était très faible avant les années 1960 malgré l'existence de la SAKAY, les départs et les arrivées étaient peu nombreux et ne concernaient qu'une minorité d'individus. Au cours de la période suivante (1961-1967), qui voit la création du BUMIDOM et le début d'une politique volontariste incitant les natifs au départ vers la métropole, le décalage entre les départs et les arrivées augmente sensiblement (-7 951 en six ans soit un solde moyen annuel de -1 300). Le solde migratoire intercensitaire va ensuite se renforcer à la fin des années 1960 (-11 719 soit une moyenne de -1674 par an). Cette période où le BUMIDOM prend en charge la quasi-totalité des départs de Réunionnais ne sera pourtant pas celle où le solde migratoire atteint son maximum. Il faut attendre la période 1974-1982 et la conjonction d'un mouvement d'émigration organisée et spontanée pour atteindre la valeur maximale du solde migratoire négatif à La Réunion. Celui-ci atteindra -33 480 personnes entre 1974 et 1982 soit un déficit net moyen de près de 4200 individus chaque année (Festy et Hamon, 1983).

22 L'idée d'une émigration de petits propriétaires réunionnais démunis est lancée dès les années 1940. Le BDPA (Bureau pour le développement de la production agricole), créé en 1950, se tourne alors vers Madagascar, afin d'y implanter les petits cultivateurs réunionnais confrontés à la pénurie de terres et à la diminution des emplois agricoles. La SAKAY (terme qui désigne un fleuve et une région situés dans le moyen ouest malgache) voit le jour en 1952 et subsistera jusqu'en 1977.

23 Créé en juin 1963, à l'instigation de Michel Debré, alors député de La Réunion, le BUMIDOM (bureau des migrations des populations d'outre-mer) est une société d'État chargée d'inciter puis d'organiser et de gérer l'émigration des natifs des DOM en direction de la métropole. Le BUMIDOM poursuit trois objectifs : il s'agit de répondre au défi de la croissance démographique, de lutter contre le chômage et de préserver la paix sociale dans les Dom. La politique du Bumidom a été « volontariste », les incitations au départ fortes.

24 En 1965, le CNARM (Centre national d'accueil des Réunionnais en métropole) est créé en appui de l'activité de la société d'État. Il est chargé d'accueillir et de favoriser l'intégration des migrants.

25 En 1982, l'agence nationale pour l'insertion et la promotion des travailleurs d'outre-mer (ANT) est créée avec pour objectif non plus d'organiser ou de favoriser les départs des Dom vers la métropole, mais plutôt de viser à l'insertion sociale et professionnelle des émigrants déjà présents sur le continent et de faciliter les retours. L'ANT centrera ses actions sur les parcours d'insertion des migrants autour du triptyque « logement-formation-emploi ».

26 Les émeutes du quartier du Chaudron, en 1991, vont à nouveau mettre en avant les difficultés de la jeunesse réunionnaise confrontée à un sous-emploi généralisé et replacer la question de l'émigration des jeunes réunionnais sur le devant de la scène politique locale et nationale. Le départ des jeunes pour une formation et/ou un emploi en métropole est à nouveau fortement encouragé par l'État et les collectivités locales. Aujourd'hui, les dispositifs de mobilité sont très nombreux ; ils permettent « une mobilité à la carte » adaptée aux besoins du plus grand nombre. 
Tableau 3 : Estimation du solde migratoire intercensitaire

\begin{tabular}{|l|c|}
\hline Population recensée au 01-07-1954 & 274370 \\
\hline Solde migratoire du 01-07-1954 au 08-10-1961 & -965 \\
\hline Population recensée au 09-10-1961 & 349282 \\
\hline Solde migratoire du 09-10-1961 au 15-10-1967 & -7951 \\
\hline Population recensée au 16-10-1967 & 416525 \\
\hline Solde migratoire du 16-10-1967 au 15-10-1974 & -11719 \\
\hline Population recensée au 16-10-1974 & 480152 \\
\hline Solde migratoire du 16-10-1974 au 08-03-1982 & -33481 \\
\hline Population recensée au 09-03-1982 & 515814 \\
\hline Solde migratoire du 09-03-1982 au 14-03-1990 & 2919 \\
\hline Population recensée au 15-03-1990 & 597823 \\
\hline Solde migratoire du 15-03-1990 au 07-03-1999 & 16271 \\
\hline Population recensée au 08-03-1999 & 706300 \\
\hline
\end{tabular}

Source : Recensements de la population et État Civil. Les soldes de la période 1954-1974 : Festy et Hamon (1983) ; 1974-1982 : données issues des publications INSEE ; calculs de l'auteur pour les périodes 1982-1999.

Le changement d'orientation de la politique de mobilité avec la fin du BUMIDOM et la création de l'ANT qui a accompagné « l'essoufflement» du nombre de départs de La Réunion vers l'extérieur au début des années 1980 va entraîner un renversement du signe du solde migratoire. Après avoir été constamment négatif de 1954 à 1982, le solde migratoire réunionnais devient positif en raison de la conjugaison de la baisse des départs et de l'augmentation des arrivées et des retours de natifs. Légèrement positif entre 1982 et $1990\left(+4000^{27}\right.$, soit 500 personnes supplémentaires par an en moyenne), le surplus des arrivées sur les départs s'est ensuite accentué entre 1990 et 1999 pour atteindre un niveau jamais atteint depuis la départementalisation (+16 300 individus soit un excédent annuel net de 1800 individus en moyenne).

Le rapprochement de la valeur de ces soldes avec le nombre d'arrivées à La Réunion au cours des périodes intercensitaires permet d'estimer le nombre de départs vers l'extérieur. La lecture des mouvements d'arrivées et de départs permet de dépasser l'analyse des soldes intercensitaires où ces flux contraires sont compensés (Graphique 1). La gestion volontariste de l'émigration par les décideurs a eu l'effet escompté. Le nombre de départs a progressivement augmenté pour atteindre un maximum de 8000 par an en moyenne entre 1974 et 1982, avant de diminuer dans la période récente. Cependant, dans le même temps le nombre de personnes venant s'installer à La Réunion, a connu une croissance régulière et soutenue.

27 Après majoration du solde de 1000 personnes supplémentaires par l'INSEE pour tenir compte du changement de comptabilisation des militaires du contingent aux recensements de 1982 et 1990. 
Graphique 1 : Les mouvements migratoires à travers les recensements

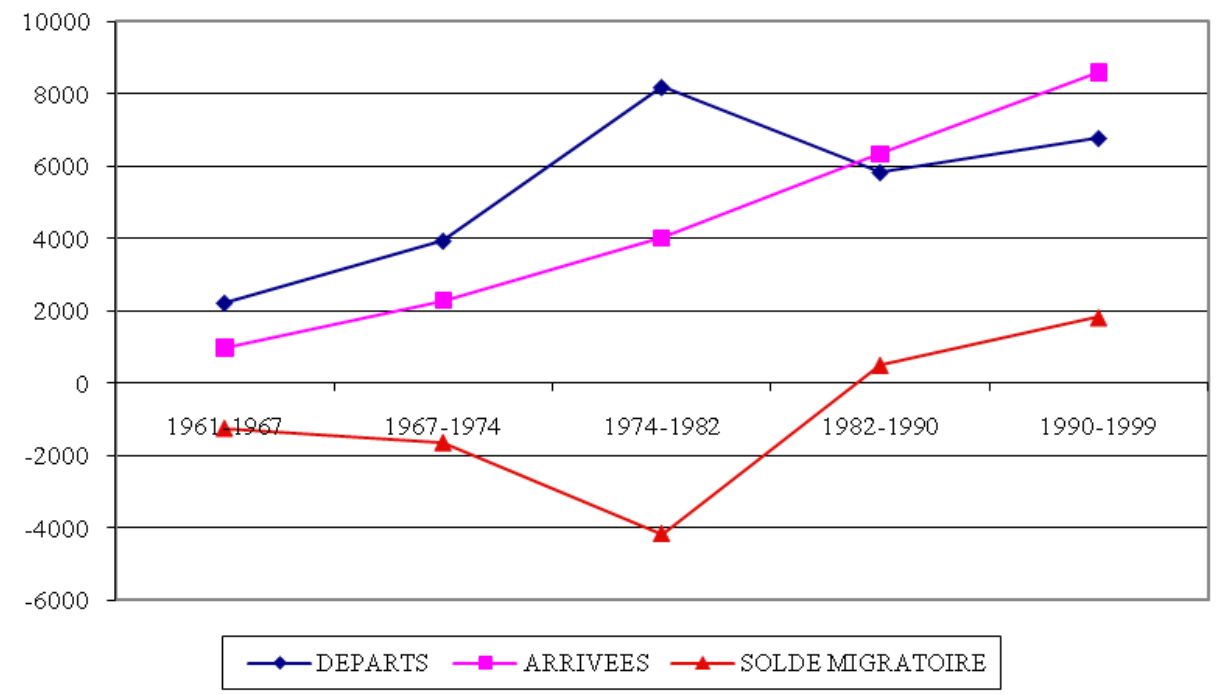

Source : INSEE, Recensements de la population et État Civil.

C'est principalement cette baisse du nombre de départs, alors que les arrivées poursuivaient leur progression, qui explique que le solde soit positif depuis 1982. La crise en métropole, associée à une moindre incitation au départ de La Réunion en raison des changements d'orientation de la politique de migration, se sont traduits par une baisse du nombre total de départs. Entre les périodes 1974-1982 et 1982-1990, le nombre moyen de départs annuel diminue de $30 \%$. Dans le même temps, le nombre des arrivées augmente encore sensiblement (6 300 arrivées chaque année en moyenne entre 1982 et 1990 contre 4000 lors de la période précédente). Au cours de la décennie 1990, qui voit la relance de la politique de mobilité par les pouvoirs publics après les émeutes du quartier du Chaudron, le nombre de départs augmente à nouveau (6 800 départs en moyenne annuelle soit une progression de $16 \%$ par rapport à la période intercensitaire précédente). Cependant, cette reprise des départs n'a pas permis de compenser la hausse plus prononcée encore du nombre des arrivées $(+36 \%)$. L'écart s'est donc creusé et le solde amplifié, l'île accueille plus d'habitants qu'elle n'en voit partir.

\section{Des mouvements de sens contraires selon le lieu de naissance des individus}

Les soldes intercensitaires résument des mouvements de sens contraire qui se compensent. Les flux opposés des natifs de La Réunion qui émigrent vers l'extérieur et des personnes nées en métropole, à l'étranger ou dans un autre Dom-Tom qui, au contraire, immigrent à La Réunion n'apparaissent pas dans le solde global. Pour mieux comprendre 
la dynamique des migrations à La Réunion, la distinction entre ces populations est pourtant nécessaire.

Depuis 1954, le solde migratoire intercensitaire estimé pour les natifs de La Réunion est constamment négatif (Graphique 2). Le nombre de départs de natifs de La Réunion a augmenté continuellement et fortement jusqu'aux débuts des années 1980 sous l'effet de la politique d'émigration impulsée par l'État sous l'égide du BUMIDOM. Le solde des natifs a ensuite fortement diminué au cours de la période suivante (-2 500 départs nets par an en moyenne), il a été divisé par 2,5. Le changement d'orientation de la politique de mobilité avec la création de l'ANT, les difficultés d'insertion accrues des migrants en métropole, mais aussi et surtout l'augmentation des retours dans le département, expliquent cette diminution du solde des natifs. Sur la dernière période, le solde net moyen s'est à nouveau renforcé, il atteint près de 3000 sorties nettes par an et retrouve ainsi le niveau du solde des années 1967-1974, c'est-à-dire au moment le plus fort de l'activité du BUMIDOM. Le solde 1990-1999 est cependant deux fois moins important que le record atteint entre 1982 et 1990, notamment en raison de la baisse du nombre de départs et de la hausse des retours.

Graphique 2 : Soldes migratoires intercensitaires sur le lieu de naissance (natifs/non natifs)

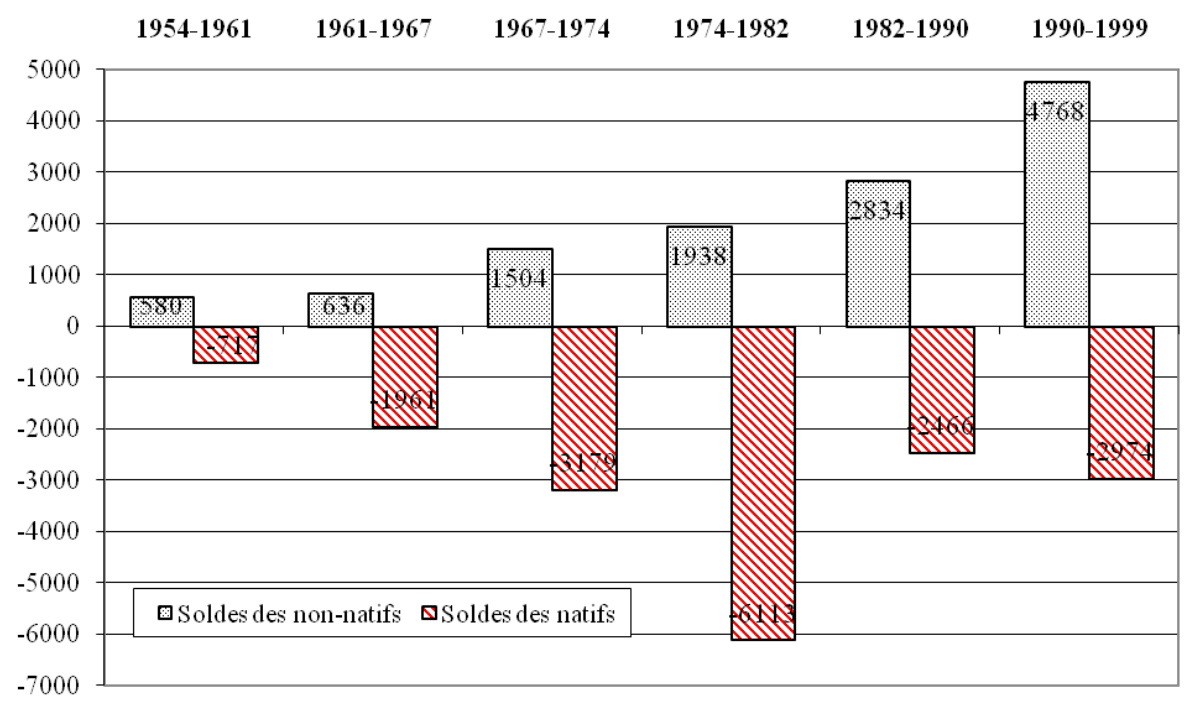

Source : INSEE, Recensements de la population et État Civil.

Pour avoir une vision d'ensemble, il faut compléter cette analyse en s'intéressant au solde des populations immigrantes à La Réunion, c'est-à-dire aux personnes nées hors de l'île venant s'y installer plus ou moins durablement. À l'inverse du solde des natifs, celui des personnes nées hors du département (en métropole, à l'étranger, dans un autre 
Dom-Tom) est positif depuis 1954. Celui-ci augmente régulièrement et fortement depuis cette date. Entre 1967 et 1974, on comptait 1500 installations nettes d'immigrants sur l'île, près de 2000 dans la période intercensitaire suivante et près de 3000 entre 1982 et 1990. Au cours de la décennie 1990, le décalage entre les arrivées et les départs de personnes nées hors de l'île s'est considérablement amplifié, il atteint un maximum proche de 4800 personnes supplémentaires par an en moyenne. Le nombre d'immigrants arrivant chaque année à La Réunion est donc plus important aujourd'hui que pendant les phases de développement accéléré des systèmes éducatifs, sociaux et de santé.

L'approche de la migration selon l'âge et le lieu de naissance entre 1990 et 1999 indique que la migration concerne très majoritairement des jeunes adultes en âge de travailler, c'est-à-dire des individus qui ont vocation à s'insérer sur le marché de l'emploi (Graphique 3).

\section{Graphique 3 : Solde migratoire selon l'âge et le lieu de naissance entre 1990 et 1999}

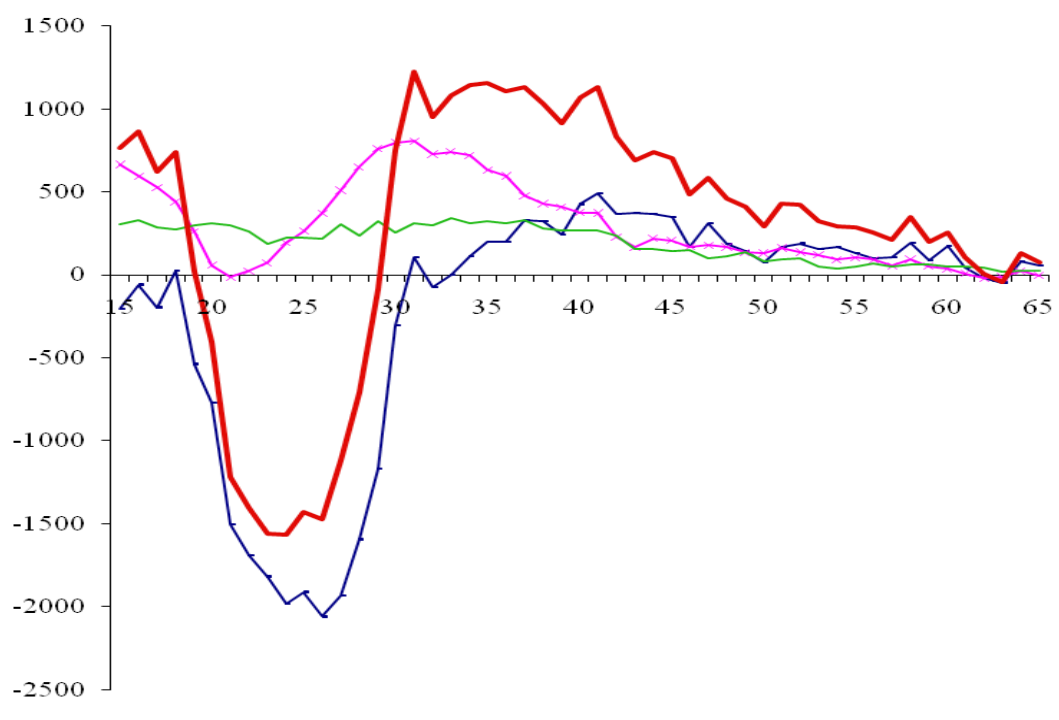

- SoldeNatifs — Soldemétro — Soldeautres $\longrightarrow$ Solde ensemble

Source : Recensements de la population et État civil ; calculs de l'auteur.

Les départs de La Réunion vers l'extérieur concernent essentiellement des jeunes adultes de dix-neuf à trente ans, alors que les arrivées se concentrent après trente ans. Le nombre des départs est toujours supérieur à celui des arrivées entre dix-neuf à trente ans alors qu'aux autres âges ce sont les arrivées qui l'emportent. Parmi l'ensemble des mouvements, seuls les natifs de La Réunion présentent un solde migratoire négatif, le solde des personnes nées hors du département est toujours positif, soulignant ainsi l'importance de l'immigration nette à tous les âges au cours de la dernière décennie. Ces mouvements de 
population ont pour effet d'augmenter les effectifs des classes d'âges au-delà de trente ans et à l'inverse de réduire les effectifs de jeunes adultes, fortement touchés par le chômage ce qui participe au vieillissement de la population.

\section{Les retours des natifs de La Réunion ayant préalablement migré}

Parallèlement à l'augmentation de la population des natifs de La Réunion en métropole, le nombre de natifs de retour de migration augmente à chaque recensement (Graphique 4). En décalage de quelques années avec le nombre maximum de départs, le nombre de retours a fortement augmenté lors de la période intercensitaire suivante (1982 et 1990). La période 1990-1999 est celle qui enregistre le nombre de retours le plus élevé. Le taux de retour des natifs de métropole est stable (23,5 p. 1000 entre 1990 et 1999 contre 22,1 entre 1982 et 1990), mais la propension au retour des natifs de La Réunion après avoir connu une migration a augmenté. Au cours de la période 1990-1999, les retours viennent plus largement compenser les départs ${ }^{28}$. Ainsi, en 1999, si on a recensé quelque 20400 Réunionnais récemment installés en métropole puisqu'ils vivaient à La Réunion en 1990 on compte à l'inverse 19800 Réunionnais qui sont revenus dans leur département après un séjour en métropole. Le solde net des mouvements de natifs entre La Réunion et la métropole sur la période est faible.

\section{Graphique 4 : Retours des natifs de La Réunion selon les périodes intercensitaires (en moyenne par an)}

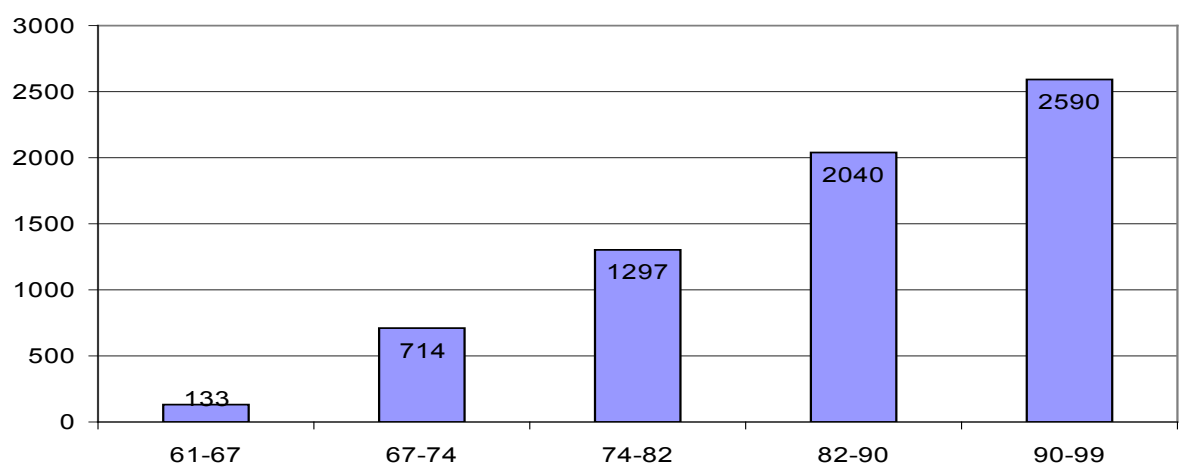

Source : INSEE, Recensements de la population.

28 D'après les données publiées par l'INSEE, la propension au retour des natifs de La Réunion de métropole s'établissait à $15 \%$ pour la période 1968-1974 et à $20 \%$ entre 1975 et 1982 (INSEE, 1987). En appliquant cette méthode de calcul aux périodes les plus récentes, on constate que la propension au retour a fortement augmenté : les retours de métropole représentent les trois quarts de la migration antérieure pour les périodes 1982-1990 et 1990-1999. Cet indicateur, plus conjoncturel que le taux de retour, permet de vérifier que les retours viennent beaucoup plus largement compenser les flux de départs que lors des périodes intercensitaires précédentes, ce qui reflète à la fois la diminution des flux de départ et l'augmentation des retours de migration. 
Le net ralentissement de la progression de la population des natifs de La Réunion en métropole ${ }^{29}$ indique qu'à la logique d'émigration de peuplement lors de la période du BUMIDOM s'est substituée peu à peu une émigration moins durable, plus marquée par la courte durée dans le cadre d'une circulation accrue entre ce département et la métropole. Cette circulation se traduit par des allers-retours plus fréquents (Marie et Temporal, 2001). En 1999, aux âges où les départs des natifs sont les plus nombreux (entre vingt-trois et vingt-sept ans), ils atteignent environ $20 \%$ d'une classe d'âge comme au cours de la période précédente. En outre, les retours après un séjour hors de l'île viennent en partie compenser l'effet des départs, notamment dans les années récentes. Au total, ces flux d'intensité variables selon les périodes et l'orientation de la politique de migration, plus ou moins incitative, restent relativement modérés en comparaison d'autres sociétés insulaires, comme dans le pacifique Sud par exemple, où l'émigration atteint des niveaux beaucoup plus hauts (Rallu, 1997b).

Enfin, du point de vue de l'immigration, on constate que les flux d'arrivée d'une population non native dans le département se sont considérablement amplifiés au cours des dernières décennies. Ainsi, malgré l'éloignement de la métropole et son isolement géographique, La Réunion connaît des flux d'entrées d'une ampleur comparable à l'ensemble des régions métropolitaines (Chevalier, 2001). Avec 11 \% d'arrivants entre 1990 et 1999 (77 400 individus recensés à La Réunion en 1999 ont déclaré résider hors de l'île en 1990), La Réunion se situe dans la moyenne des régions françaises qui est de 11,6 \%.

La thèse du «transfert démographique » de population, de La Réunion vers l'extérieur, et notamment d'individus en âge de travailler, est mise à mal par cette analyse des flux. Entre 1974 et 1982, les départs avaient permis de limiter l'accroissement démographique de moitié environ (Rallu, 1997a : 700), les migrations exerçant un effet négatif sur les effectifs de population du département. L'effet est inverse aujourd'hui en raison de la hausse des retours et des arrivées qui se traduit par un solde positif. Si l'apport migratoire reste modéré au regard de l'accroissement naturel (la migration explique $15 \%$ de la croissance démographique entre 1990 et 1999), le renversement du signe du solde migratoire et l'augmentation des arrivées tiennent une place importante dans l'opinion publique, principalement en raison de ses incidences sur le marché de l'emploi. La structure par âge des immigrants, majoritairement actifs, crée des tensions sur un marché de l'emploi saturé et sélectif.

\section{L'IMPACT DE LA MIGRATION SUR LA POPULATION ACTIVE}

\section{Un impact « macro » modéré...}

Au recensement de 1999, on dénombre un total de 98000 immigrants résidant à La Réunion pour 608160 natifs (Tableau 4). Les immigrants représentent ainsi près de $14 \%$ de la population totale de l'île. La part des immigrants dans la population de l'île

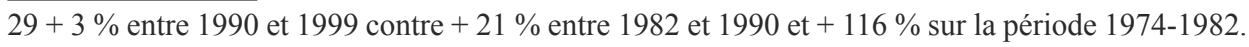


augmente régulièrement, elle a doublé entre les recensements de 1982 et $1999^{30}$. Parmi ces immigrants, les personnes nées en métropole sont nettement majoritaires, à eux seuls ils représentent les deux tiers des immigrants en 1999, soit 9,1 \% de la population de l'île. Les personnes nées à l'étranger ou dans un autre Dom-Tom représentent quant à elles près de $5 \%$ de la population résidant à La Réunion en $1999^{31}$.

Tableau 4 : Population résidant à La Réunion en 1999 selon le lieu de naissance

\begin{tabular}{|l|c|c|}
\hline & Effectifs & $\begin{array}{c}\text { Part } \\
\text { en } \%\end{array}$ \\
\hline POPULATION RÉSIDANT À LA RÉUNION & $\mathbf{7 0 6} \mathbf{1 8 0}$ & $\mathbf{1 0 0}$ \\
Nés à La Réunion & $\mathbf{6 0 8} \mathbf{1 5 7}$ & $\mathbf{8 6 , 1}$ \\
Nés hors de La Réunion dont : & $\mathbf{9 8} \mathbf{0 2 3}$ & $\mathbf{1 3 , 9}$ \\
$\quad$ Nés en métropole & 64561 & 9,1 \\
$\quad$ Nés dans un autre DOM-TOM & 9114 & 1,3 \\
$\quad$ Nés à l'étranger & 24348 & 3,4 \\
\hline Population native de La Réunion résidant en métropole (1) & 94585 & - \\
ENSEMBLE DES NATIFS (à La Réunion et en métropole) (2) & 702742 & - \\
Part des Réunionnais de métropole sur l'ensemble des natifs $\left[(1) /(2)^{*} 100\right]$ & - & $\mathbf{1 3 , 5}$ \\
\hline
\end{tabular}

Source : INSEE, Recensement de la population ; calculs de l'auteur.

À la même date, on dénombre 94585 natifs de La Réunion recensés sur le territoire métropolitain, soit une population presque équivalente à celle des immigrants à La Réunion. Ainsi, en 1999, l'impact net de la migration sur les effectifs de population est quasiment nul, le nombre total d'émigrants étant très proche de celui de l'ensemble des immigrants ${ }^{32}$.

$30 \mathrm{Au}$ recensement de mars 1982, les immigrants représentaient 6,9\% de la population résidant à La Réunion, les individus nés en métropole représentaient à eux seuls $4,1 \%$ de la population totale. Ces parts étaient respectivement de $2,3 \%$ et $0,4 \%$ au recensement d'octobre 1946 , au lendemain de la départementalisation.

31 L'immigration à La Réunion concerne principalement des individus ayant des attaches fortes avec la France. Il s'agit en grande majorité d'individus nés en métropole, en outre-mer ou dans les anciennes colonies françaises. L'approche de la nationalité de ces immigrants permet d'ailleurs de constater que la très grande majorité des personnes nées à l'Étranger possèdent la nationalité française. Au recensement de 1999, si environ un quart des immigrants est né dans un pays étranger, seulement 3,6\% ont la nationalité étrangère. La Réunion est la région française qui compte la plus faible part d'étrangers recensés sur son territoire $(0,5 \%)$.

$32 \mathrm{Ne}$ disposant pas d'information sur les natifs de La Réunion vivant à l'étranger nous limitons notre analyse à la population des émigrants en métropole. Il est très difficile d'estimer les effectifs de Réunionnais résidant dans un pays étranger à partir des sources existantes, cependant, même si le départ pour une destination étrangère a pu se développer dans les années les plus récentes, les effectifs restent réduits en comparaison de la population des natifs en métropole. 
L'analyse des rapports de dépendance des immigrants recensés à La Réunion en 1999 indique que l'immigration concerne très principalement des personnes en âge de travailler (Tableau 5).

Tableau 5 : Rapports de dépendance ${ }^{33}$ des populations migrantes et non migrantes en 1999

\begin{tabular}{|l|c|c|c|}
\hline \multirow{2}{*}{ Lieu de naissance } & \multicolumn{3}{c|}{ Rapports de dépendance } \\
\cline { 2 - 4 } & Hommes & Femmes & Total \\
\hline Nés à La Réunion & 54,3 & 55,0 & 54,6 \\
\hline Nés en métropole & 35,0 & 37,9 & 36,3 \\
\hline Nés dans un autre DOM-TOM & 78,2 & 63,5 & 70,1 \\
\hline Nés à l'étranger & 21,1 & 19,1 & 20,1 \\
\hline Ensemble Réunion & $\mathbf{5 1 , 0}$ & $\mathbf{5 1 , 9}$ & $\mathbf{5 1 , 4}$ \\
\hline Natifs de La Réunion en métropole & 13,9 & 16,3 & 15,2 \\
\hline Ensemble des natifs (à La Réunion et en métropole) & $\mathbf{4 7 , 5}$ & $\mathbf{4 8 , 1}$ & $\mathbf{4 7 , 8}$ \\
\hline
\end{tabular}

Source : INSEE, Recensement de la population 1999 ; calculs de l'auteur.

La présence des immigrants sur l'île, et notamment les personnes nées en métropole et à l'étranger, concentrées aux âges d'activité (les rapports de dépendance sont respectivement de 36,3 et 20,1), vient augmenter le nombre des personnes en âge de travailler et entraîne une diminution de plus de 3 points du rapport de dépendance de l'ensemble de la population résidant à La Réunion en 1999 (51,4). L'émigration des natifs de La Réunion en métropole a eu quant à elle l'effet inverse, en ponctionnant très prioritairement les effectifs de jeunes adultes en âge de travailler. Le rapport de dépendance de la population des natifs de La Réunion en métropole indique très nettement la sélection opérée par la migration dans les effectifs d'actifs : on compte seulement 15,2 personnes âgées de moins de quinze ans ou de soixante-cinq ans et plus pour 100 individus de quinze à soixante-quatre ans. Les émigrants réunionnais sont donc très majoritairement concentrés aux âges d'activité, illustrant clairement une migration de travailleurs ou de personnes ayant vocation à travailler. Au niveau macro et à la date du recensement 1999, la migration a donc contribué à réduire, même faiblement, les effectifs de la population en âge de travailler résidant à La Réunion, diminuant la pression sur l'emploi. Cependant, cet impact global après plus de trente ans de politique d'émigration incitant les Réunionnais au départ reste très modéré, pour ne pas dire faible. La hausse du nombre des immigrants installés sur l'île et des retours de natifs au cours des dernières décennies étant venue compenser l'effet de l'émigration.

33 Nombre de personnes âgées de moins de quinze ans et de plus de soixante-quatre ans rapporté à la population des quinze/soixante-quatre ans. 


\section{...mais une recomposition sociale importante}

Ces mouvements et ces installations de populations aux profils très différents ont un impact important sur la recomposition de la population à certains niveaux de diplômes, notamment chez les plus diplômés.

Ainsi, si dans l'ensemble de la population âgée de quinze à soixante-quatre ans non scolarisée résidant à La Réunion on compte $84,8 \%$ natifs de La Réunion pour 15,2\% non natifs, ce ratio varie fortement selon le niveau de diplôme des individus. Les non diplômés sont très majoritairement des natifs de La Réunion (93,7 \%). Plus le diplôme s'élève et plus la part des immigrants, principalement ceux nés en métropole, est importante et donc plus celle des natifs se réduit (Graphique 5). Les proportions d'immigrants sont toujours très nettement supérieures à la moyenne $(15,2 \%)$ pour les catégories de diplôme équivalentes ou supérieures au bac (27,6 \% d'immigrants au niveau bac, dont $19,3 \%$ sont nés en métropole, $42,2 \%$ au niveau bac +2 , dont $32,3 \%$ nés en métropole). Au niveau de diplôme le plus élevé $(>$ bac +2$)$, les immigrants sont même plus nombreux que les natifs de La Réunion, on compte près de 14000 immigrants, dont 10500 nés en métropole pour 8600 natifs. À ce niveau de formation, la part des personnes nées hors du département atteint $62 \%$ des quinze à soixante-quatre ans ce qui illustre l'importance de l'immigration des personnes les plus qualifiées à La Réunion.

\section{Graphique 5 : Répartition des individus selon leur lieu de naissance par catégorie de diplôme (en \%)}

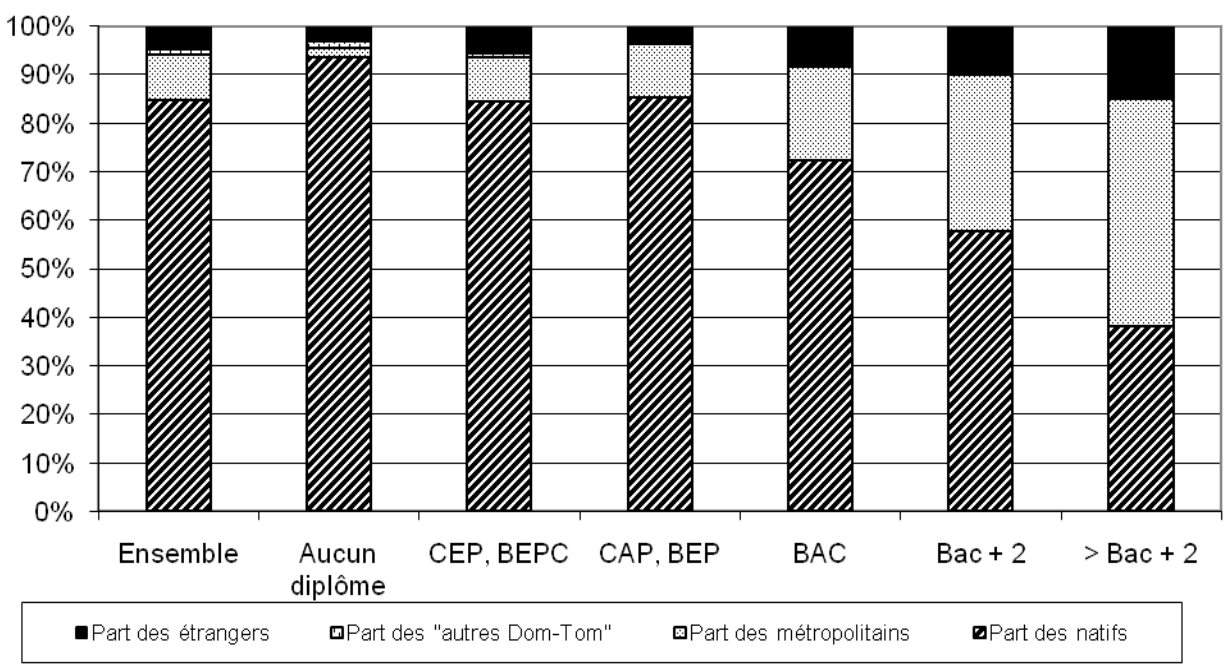

Source : INSEE, Recensement de la population; calculs de l'auteur. 
Sans les immigrants présents sur l'île en 1999, la part des individus non diplômés d'âge actif (quinze à soixante-quatre ans) passerait de 54,1 \% à 59,8\%, elle augmenterait donc de plus de 5 points. À l'autre extrême, la part des individus ayant un diplôme équivalent ou supérieur à bac +2 serait divisée de moitié passant de $10 \%$ à 5,6 \% de la population non scolarisée en âge de travailler du département. L'aperçu de ces valeurs en volume montre à quel point l'immigration de personnes très qualifiées est une réalité. Sans immigration, le nombre d'individus âgés de quinze à soixante-quatre ans non scolarisés ayant un diplôme équivalent ou supérieur au bac serait réduit de 31000 , soit une diminution de plus de $40 \%$ de la population correspondante résidant à La Réunion en 1999. L'arrivée d'immigrants et de natifs de retour plus qualifiés que les natifs non migrants a un effet positif sur la structure des diplômes de l'île. La migration permet d'élever le niveau de qualification de la main-d'œuvre dans le département.

La migration exerce également un effet sur la répartition des individus selon leur statut d'activité. Si dans l'ensemble des personnes âgées de quinze à soixante-quatre ans résidant à La Réunion en 1999 on compte une proportion de l'ordre de 16 personnes nées hors du département pour 100 natifs, ce ratio varie sensiblement selon la situation d'activité des individus. En 1999, les 73000 immigrants en âge de travailler occupent près de 36000 emplois. Le rapport est donc proche d'un emploi pour deux immigrants contre un peu plus d'un emploi pour trois natifs en âge de travailler (393 300 natifs en âge de travailler occupent 137000 emplois). Les immigrants représentent ainsi une personne en âge de travailler (quinze à soixante-quatre ans) sur six, ils occupent cependant un emploi sur cinq (20,6 \% des actifs occupés) et ne représentent qu'un chômeur sur dix (10,3\% des chômeurs).

Ainsi, la migration et ses conséquences tiennent une place importante dans l'opinion et le débat public, car elle concerne principalement des personnes en âge de travailler et entraîne une recomposition sociale importante de la population du département selon l'origine dans un contexte de chômage de masse et donc de concurrence entre individus sur le marché de l'emploi.

Il convient d'approfondir ces analyses en se demandant quel est l'impact de ces mouvements de population sur l'emploi appréhendé du point de vue des individus. La migration au départ, au retour ou à l'arrivée constitue-t-elle un atout, un avantage ou au contraire un désavantage en matière d'insertion professionnelle et de qualification des emplois occupés?

\section{MIGRATION ET EMPLOI : QUELAVANTAGE DU DÉPART POUR LES NATIFS ?}

\section{Le départ pour la métropole permet aux natifs d'accéder plus largement à l'emploi (...)}

Quelle que soit la période d'émigration des Réunionnais, en métropole leur situation vis-à-vis de l'emploi est beaucoup plus favorable que celle des natifs restés dans 
leur département d'origine. Les natifs de la Réunion en métropole ont des taux d'emploi nettement plus élevés que les non migrants et des taux de chômage beaucoup plus faibles. Les différentiels sont sensibles chez les hommes comme chez les femmes. Pour les hommes, les taux d'emploi des natifs non migrants sont inférieurs de 35 points à ceux des migrants les plus anciens et de 13 points à ceux des migrants de la période 1990-1999. Les écarts sont respectivement de 30 et 10 points chez les femmes. La différence de taux de chômage est également très marquée : les natifs non migrants affichent des taux de chômage supérieurs de 31 points aux émigrants installés avant 1990 et de 26 points aux migrants les plus récents. Pour les femmes, l'écart est également important avec les migrantes les plus anciennes (31 points), mais il est plus réduit avec les migrantes de la décennie 1990 (13 points), signe que l'insertion professionnelle des migrantes en métropole de la dernière période est plus difficile et donc plus proche de la situation observée à La Réunion. Les natifs de La Réunion qui étaient déjà installés en métropole avant 1990 ont ainsi une situation d'emploi très proche de celle de l'ensemble des métropolitains, ils se sont bien insérés et sont très majoritairement actifs occupés. Les migrants les plus récents semblent, quant à eux, éprouver plus de difficultés d'insertion, notamment les femmes.

\section{Graphique 6 : Taux d'emploi comparés des natifs installés en métropole et des non migrants selon le groupe d'âge (hommes)}

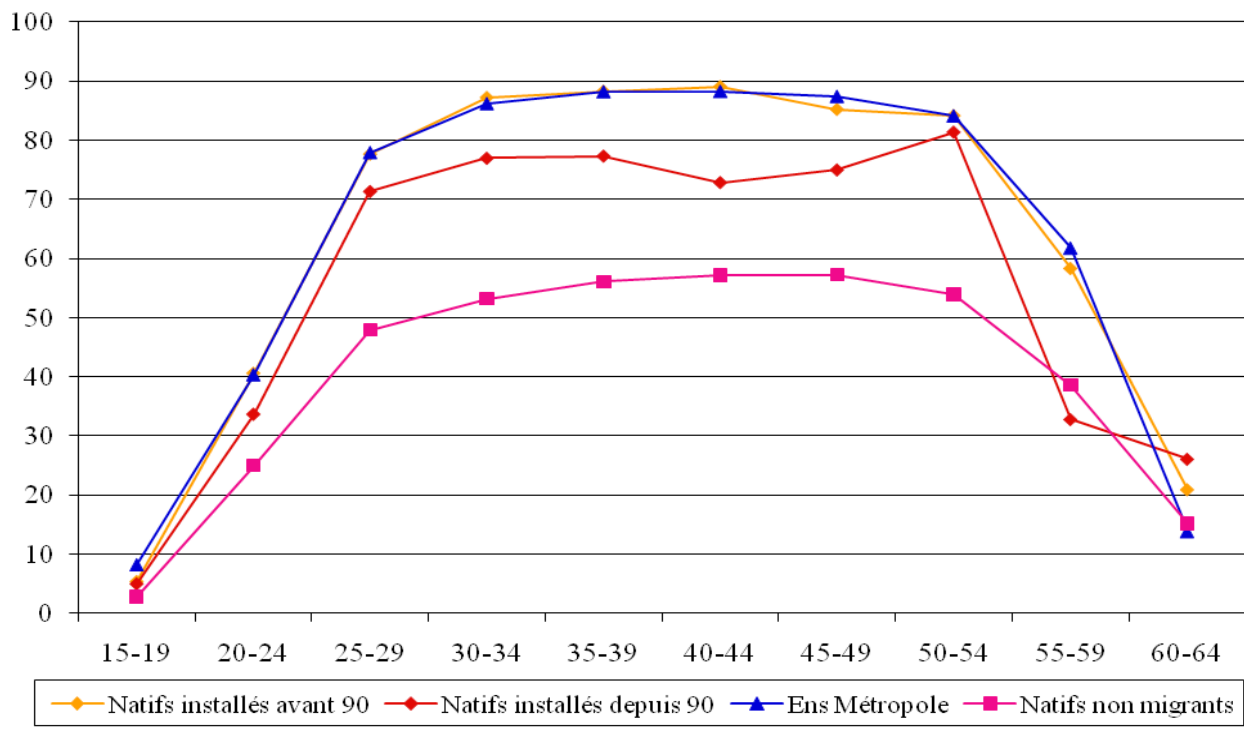

Source : INSEE, Recensements de la population 1999 Réunion et métropole ; calculs de l'auteur. 


\section{Graphique 7 : Taux d'emploi comparés des natives installées en métropole et des non migrantes selon le groupe d'âge (femmes)}

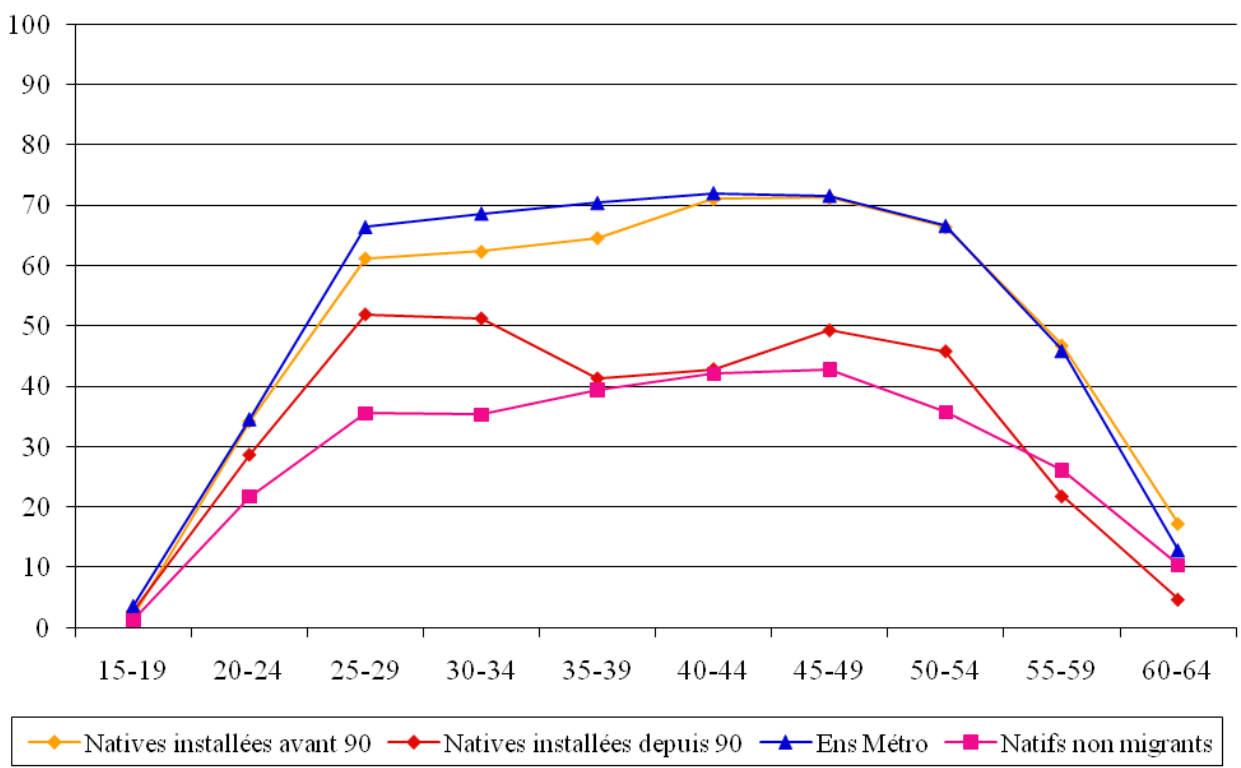

Source : INSEE, Recensements de la population 1999 Réunion et métropole ; calculs de l'auteur.

Les tendances selon l'âge des individus (Graphique 6 et 7) indiquent que le départ pour la métropole constitue un avantage d'autant plus grand en termes d'accès à l'emploi qu'il se fait jeune. Les natifs de La Réunion partis en métropole au cours de la décennie 1990 éprouvent plus de difficultés pour accéder à l'emploi en métropole entre trente-cinq et quarante-cinq ans, en particulier chez les femmes pour lesquelles le fait d'avoir migré ne semble pas permettre un accès à l'emploi plus large que si elles étaient restées dans leur département.

Compte tenu de la relation étroite entre le niveau de diplôme et les possibilités d'accès à l'emploi, il est nécessaire d'approfondir cette analyse en raisonnant à diplôme égal ${ }^{34}$.

Chez les hommes, les différentiels d'accès à l'emploi entre les Réunionnais en métropole et les natifs non migrants sont relativement faibles au niveau de diplômes les plus élevés (à partir du baccalauréat) alors qu'ils sont très marqués pour les catégories de diplômes inférieurs (Graphique 8). La différence de taux d'emploi est maximum entre les natifs installés en métropole avant 1990 et les non migrants pour les catégories de

34 Afin de s'affranchir des biais liés aux personnes en cours d'études au moment du recensement qui viennent perturber les analyses des taux d'emploi, cette approche du lien entre niveau de diplôme et emploi ne concerne que la population ayant terminé sa scolarité au moment du recensement. 
diplômes inférieurs au bac : seulement un tiers des non migrants sans diplôme occupent un emploi contre $70 \%$ des natifs installés en métropole avant 1990 et $57 \%$ de ceux partis au cours de la dernière décennie. La différence d'accès à l'emploi entre migrants et non migrants est ainsi très marquée chez les moins diplômés, les taux d'emploi des migrants les plus anciens pouvant être jusqu'à deux fois plus hauts que ceux des non migrants.

L'avantage de l'émigration pour les natifs s'atténue au fur et à mesure que le niveau de diplôme s'élève, mais il reste important jusqu'au niveau BEP. Pour les catégories de diplômes supérieurs au bac, les situations d'emploi des migrants et des non migrants sont proches et les écarts plus réduits.

Graphique 8 : Taux d'emploi comparés des natifs installés en métropole et des non migrants selon le diplôme (hommes de quinze à soixante-quatre ans non scolarisés, en \%)

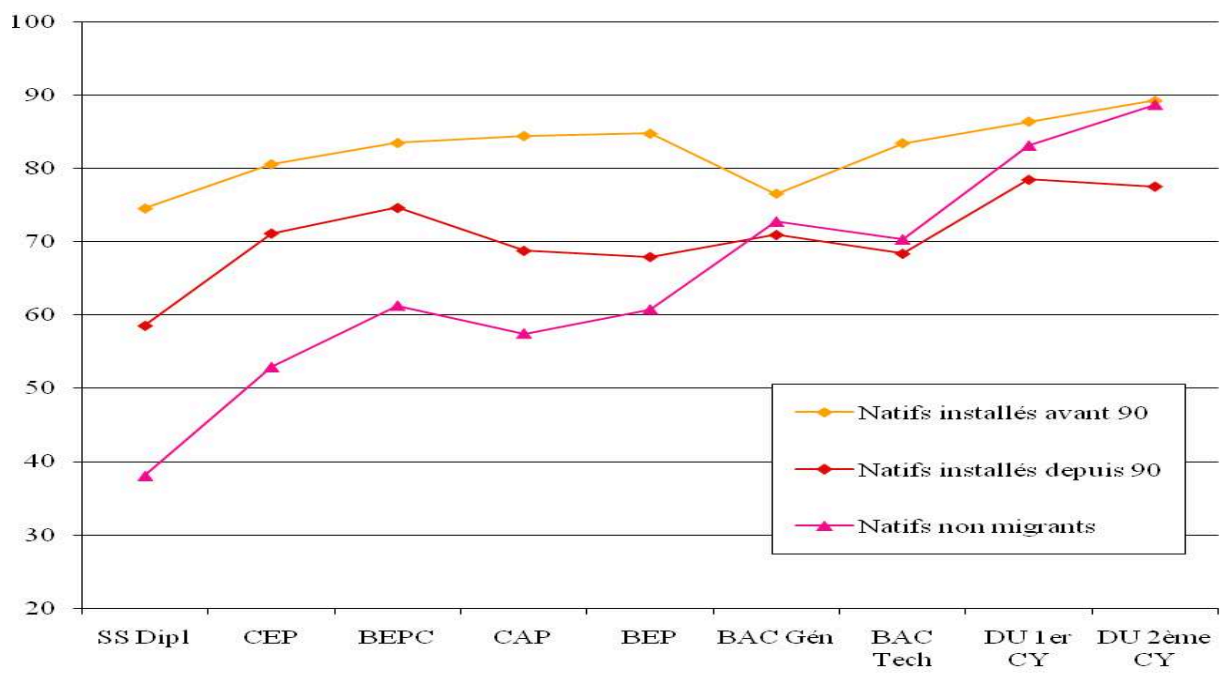

Source : INSEE, Recensements de la population 1999 Réunion et métropole ; calculs de l'auteur.

On observe globalement les mêmes tendances chez les femmes (Graphique 9), les différentiels de taux d'emploi selon le niveau de diplôme sont nets pour les niveaux de diplôme les plus bas, mais l'impact de la migration est plus réduit dans les catégories de diplôme supérieures et pour les migrantes de la décennie 1990. 
Graphique 9 : Taux d'emploi comparés des natifs installés en métropole et des non migrants selon le diplôme (femmes de quinze à soixante-quatre ans non scolarisés, en \%)

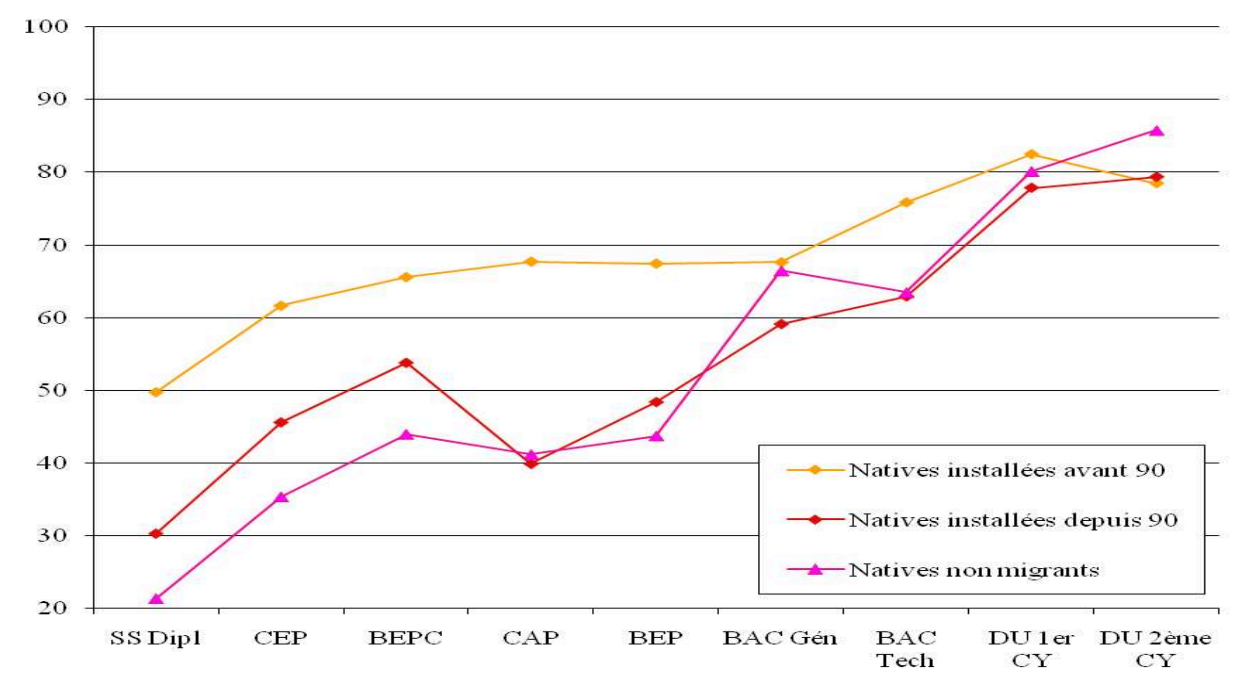

Source : INSEE, Recensements de la population 1999 Réunion et métropole ; calculs de l'auteur.

Ainsi, appréhendée à partir des taux d'emploi, la situation d'emploi des Réunionnais de métropole est plus favorable que celle des natifs restés dans leur département. L'émigration pour la métropole leur permet d'accéder plus largement à l'emploi. Le marché de l'emploi de métropole offre plus de possibilités d'insertion. En outre, la sélection opérée par la migration auprès des natifs les plus qualifiés et la différence de niveau de diplôme de ces populations expliquent une grande partie des écarts, mais certains se maintiennent à caractéristiques équivalentes. L'avantage de la migration pour la métropole se vérifie notamment pour les hommes et les moins diplômés. Les natifs de La Réunion qui ont migré en métropole au cours de la dernière décennie éprouvent plus de difficultés d'insertion que les migrants dont l'installation a eu lieu avant 1990, ces derniers se sont bien insérés sur le territoire métropolitain, leurs taux d'emploi sont équivalents à l'ensemble de la population de métropole.

\section{(...) les natifs de La Réunion en métropole occupent néanmoins des emplois moins qualifiés que la moyenne métropolitaine et que les non migrants}

S'ils accèdent plus largement aux emplois, les natifs de La Réunion en métropole sont cependant surreprésentés dans les catégories " ouvriers " et "employés " au détriment des catégories supérieures. Plus des trois quarts des hommes et des femmes qui occupent un emploi en métropole sont classés dans ces catégories. Les hommes sont plus souvent « ouvriers » (près de la moitié des natifs de La Réunion de métropole) alors que 
les femmes ont plus souvent le statut d' « employé » (plus des deux tiers des natives ayant un emploi). Ces proportions d'ouvriers et d'employés sont plus élevées que la moyenne des travailleurs de métropole $(50 \%$ des hommes et $60 \%$ des femmes sont classés dans l'une ou l'autre de ces catégories) et que celle observée à La Réunion (65\% des hommes et $71 \%$ des femmes).

En outre, parmi les salariés (public et privé) la qualification des emplois occupés par les natifs de La Réunion les plus anciennement installés en métropole est relativement proche de celle des non migrants, quel que soit le niveau de diplôme et le sexe. Les Réunionnais les plus récemment installés occupent par contre des emplois nettement moins qualifiés, ils accèdent en très faibles proportions aux emplois des catégories supérieures et cela même s'ils ont des niveaux de diplôme élevés ce qui confirme les difficultés accrues des migrants les plus récents.

Enfin, la comparaison avec la moyenne métropolitaine indique des différences de qualification des emplois occupés très nettes. Les natifs de La Réunion en métropole occupent des emplois toujours moins qualifiés que l'ensemble des métropolitains à niveau de diplôme égal. Les écarts sont prononcés chez les hommes comme chez les femmes à chaque niveau de diplôme. Par exemple, parmi les hommes titulaires d'un CAP ou d'un BEP, 24,1 \% des métropolitains occupent un emploi en tant que « cadres » ou « professions intermédiaires » contre seulement $10 \%$ des natifs installés en métropole arrivés après 1990 et 14,6\% de ceux installés avant cette date. Ces proportions sont respectivement de $14,6 \%, 7,1 \%$ et $8,6 \%$ chez les femmes. Chez l'ensemble des salariés titulaires d'un diplôme de second ou troisième cycle de l'enseignement supérieur, 77,1 \% des hommes de métropole sont « cadres supérieurs » contre seulement 49,4\% des natifs installés après 1990 et 68,5 \% de ceux dont l'installation est plus ancienne. Ces écarts sont du même ordre chez les femmes avec néanmoins des proportions plus basses, car elles sont moins nombreuses que les hommes à accéder aux emplois les plus qualifiés (respectivement : $55,9 \%, 34,1 \%$ et $40 \%)$.

Ces écarts importants à niveau de diplôme équivalent indiquent que les émigrants réunionnais semblent désavantagés sur le marché du travail de métropole, ils sont plus nombreux en poids relatif à occuper des emplois à faible niveau de qualification. Les émigrants de la dernière décennie sont ceux qui éprouvent le plus de difficultés. À l'exception des natifs de La Réunion les plus diplômés, groupe au sein duquel les émigrants accèdent plus largement aux emplois de «cadres supérieurs » que les non migrants, le départ pour la métropole ne leur permet pas d'accéder à des emplois de niveau de qualification plus haut que les natifs restés dans leur département. 


\section{MIGRATION ET EMPLOI : QUEL AVANTAGE AU RETOUR ET À L'ARRIVÉE À LA RÉUNION?}

\section{Les natifs de retour accèdent plus largement à l'emploi que les non migrants, mais cependant moins que les immigrants nés en métropole}

Du point de vue de La Réunion, les immigrants nés en métropole et les natifs de retour de migration ont des taux d'emploi supérieurs et des taux de chômage inférieurs aux non migrants qui ont la situation d'emploi la plus défavorable ${ }^{35}$.

Les natifs de retour parviennent ainsi à tirer un bénéfice de leur expérience de migration sur le marché du travail de l'île. L'avantage des métropolitains est plus prononcé encore, la différence de taux d'emploi avec les non migrants est nette, à tous les âges. Les écarts sont limités jusqu'à trente ans puis se stabilisent ensuite à un niveau élevé (Graphique 10). Entre trente et cinquante ans, les taux d'emploi des hommes nés en métropole oscillent de $77 \%$ à $85 \%$ selon la période de leur installation et leur âge alors qu'ils varient de $53 \%$ à $62 \%$ chez les natifs de La Réunion selon qu'ils soient non migrants ou natifs de retour, soit une différence de plus de 20 points selon le lieu de naissance.

Graphique 10 : Taux d'emploi comparés des natifs de retours, des immigrants nés en métropole et des non migrants selon le groupe d'âge (hommes, en \%)

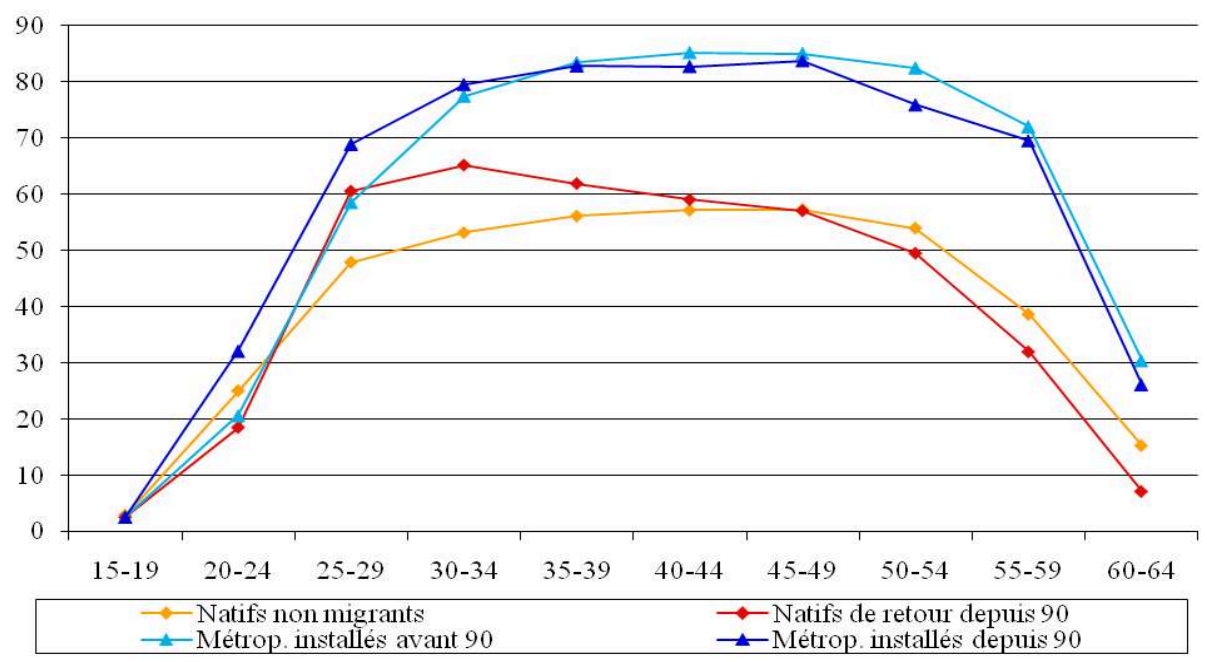

Source : INSEE, Recensements de la population 1999 Réunion ; calculs de l'auteur.

35 Avec les personnes nées dans un Tom et notamment à Mayotte qui ont des taux d'emploi extrêmement faibles. La grande majorité des originaires de Mayotte qui se portent sur le marché de l'emploi réunionnais sont au chômage. Soulignons que le 31 mars 2011, Mayotte est devenue officiellement le 101e département français. 
Graphique 11 : Taux d'emploi comparés des natives de retours, des immigrantes nées en métropole et des non migrantes selon le groupe d'âge (femmes, en \%)

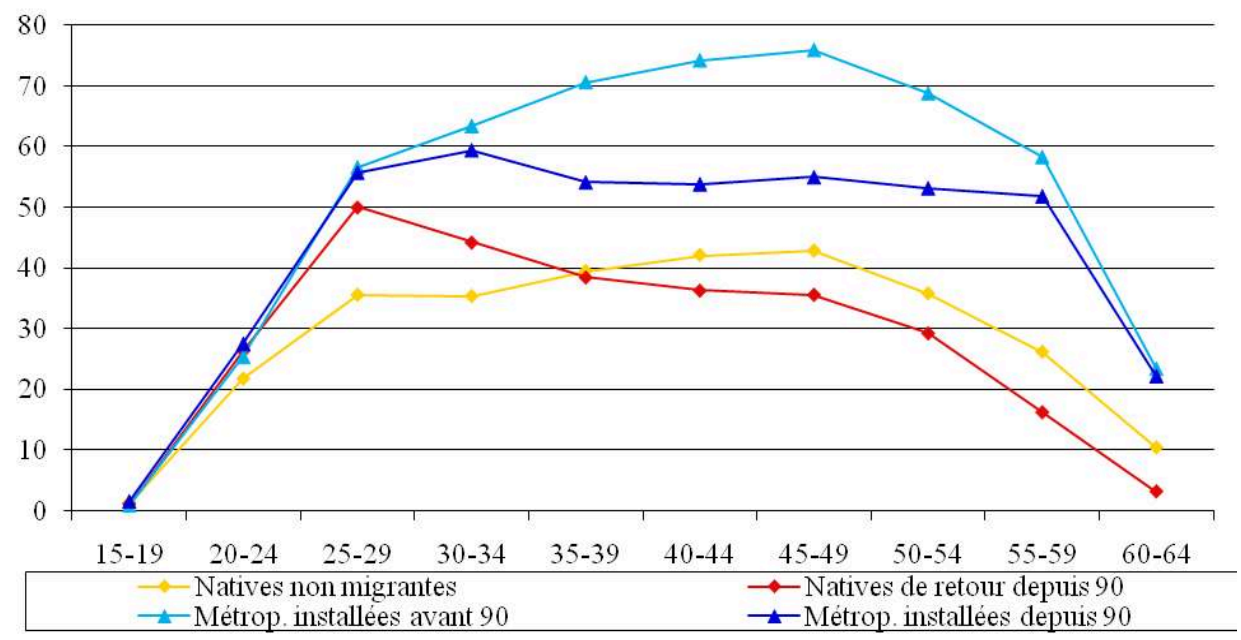

Source : INSEE, Recensements de la population 1999 Réunion ; calculs de l'auteur.

Les natifs de La Réunion de retour dans le département après 1990 ont des taux d'emploi supérieurs aux natifs n'ayant pas migré, mais seulement dans les classes d'âges les plus jeunes. On remarque en effet que le bénéfice du retour par rapport aux non migrants n'est sensible que pour les natifs de retour de moins de quarante ans. Après cet âge, le fait d'être de retour de migration n'est plus un avantage sur le marché du travail de l'île, il peut même devenir un handicap en fin de vie active, les natifs de retour occupant alors toujours moins souvent un emploi que les non migrants. Les tendances sont similaires chez les femmes (Graphique 11), les taux d'emploi des femmes nées en métropole sont nettement supérieurs à ceux des natives de La Réunion, migrantes et non migrantes. L'avantage du retour pour les natives ne s'observe que pour les classes d'âge les plus jeunes, après quarante ans les taux d'emploi des natives de retour sont inférieurs à ceux des natives n'ayant pas fait le choix de la migration. Le retour après une migration est donc un avantage s'il a lieu en début de vie active. Enfin, à la différence des hommes, les immigrantes nées en métropole et arrivées au cours de la dernière décennie semblent éprouver plus de difficultés d'accès à l'emploi que leurs homologues dont l'installation est plus ancienne.

La différence de niveau de diplôme de ces populations explique une grande partie de ces écarts. À diplôme égal, l'avantage de la migration des arrivants sur l'île est plus limité ${ }^{36}$.

36 Comme précédemment et afin de s'affranchir des biais liés à la population en cours d'études au moment du recensement nous présentons ici les taux d'emploi des populations migrantes et non migrantes ayant terminé leur scolarité en 1999. 
Graphique 12 : Taux d'emploi comparés des natifs de retours, des immigrants nés en métropole et des non migrants selon le niveau de diplôme (hommes de quinze à soixante-quatre ans non scolarisés, en \%)

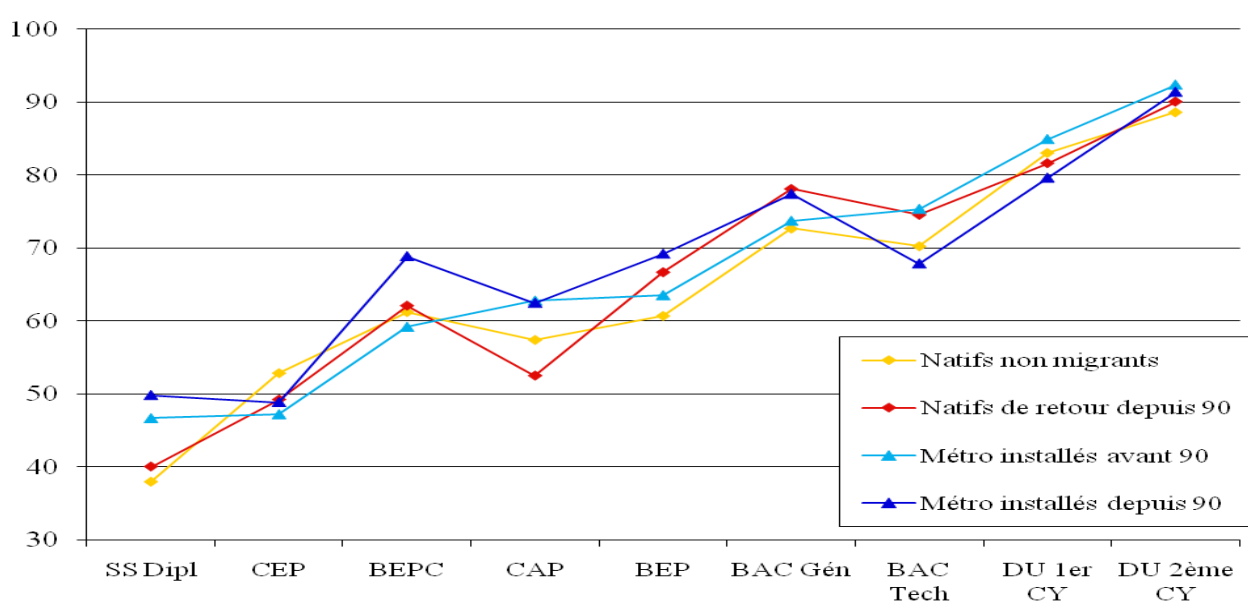

Source : INSEE, Recensement de la population 1999, Réunion ; calculs de l'auteur.

Chez les hommes, les natifs de La Réunion de retour de migration et les métropolitains arrivés après 1990 bénéficient des taux d'emploi les plus élevés. C'est vrai pour les niveaux de diplôme les plus bas (sans diplôme, CEP, BEPC), mais également pour les titulaires d'un baccalauréat général ou technique (Graphique 12).

Quel que soit le niveau de diplôme (à l'exception du CAP) les natifs de La Réunion de retour de migration ont des taux d'emploi supérieurs aux natifs non migrants, mais les écarts sont réduits (de 1 à 7 points de différence selon le niveau de diplôme). Ainsi, pour les individus sans diplôme ayant terminé leur scolarité qui réunissent les effectifs les plus nombreux, $38 \%$ des natifs de retour occupent un emploi contre $33 \%$ des non migrants. Pour les diplômés de l'enseignement supérieur, les courbes des taux d'emploi des différentes populations sont très proches, les différences sont faibles. 
Graphique 13 : Taux d'emploi comparés des natives de retours, des immigrantes nées en métropole et des non migrantes selon le niveau de diplôme

(femmes de quinze à soixante-quatre ans non scolarisés, en \%)

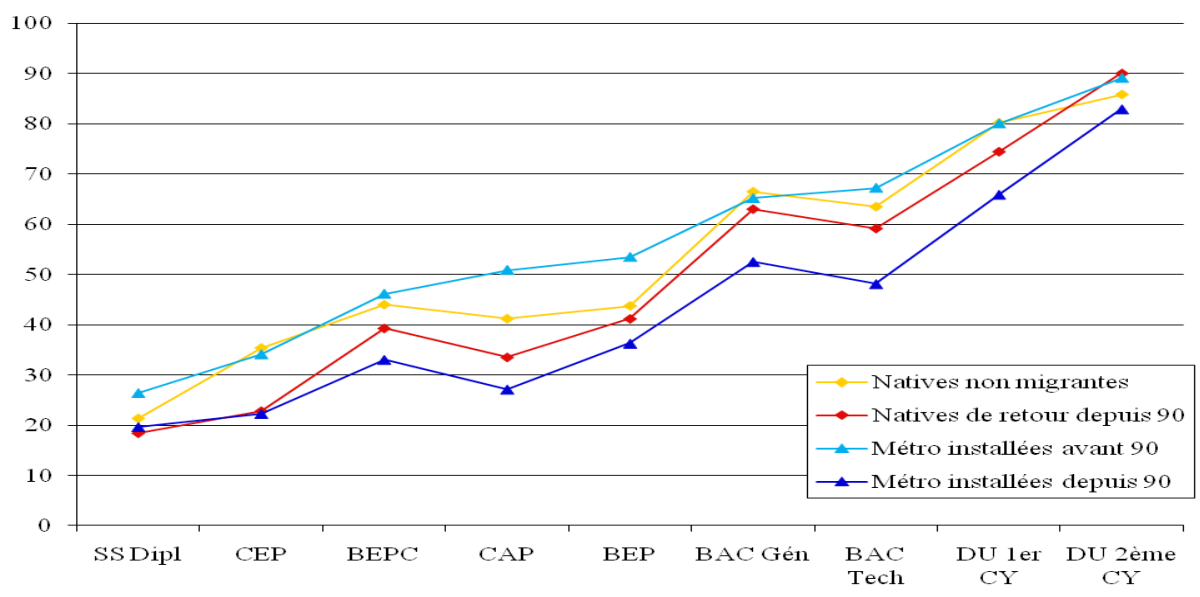

Source : INSEE, Recensement de la population 1999, Réunion ; calculs de l'auteur.

Pour les femmes (Graphique 13), l'avantage de l'origine métropolitaine ou du retour de métropole sur le marché de l'emploi doit également être relativisé à diplôme égal. Quel que soit le niveau de diplôme, les métropolitaines arrivées à La Réunion après 1990 ont toujours des taux d'emploi inférieurs aux autres catégories de population. On observe la même tendance pour les natives de La Réunion revenues dans leur département entre 1990 et 1999. Elles ont des taux d'emploi toujours plus faibles que les natives non migrantes, avec cependant une exception notable pour les plus diplômées de retour qui occupent proportionnellement plus souvent un emploi que les non migrantes et que les métropolitaines. À ce niveau de diplôme le plus élevé, les natives de La Réunion de retour après une migration semblent tirer un bénéfice de leur séjour à l'extérieur, elles accèdent plus à l'emploi que les non migrantes.

En se centrant sur l'analyse des générations âgées de vingt-cinq à trente-neuf $a^{37}$ afin de limiter l'effet de l'âge, les différentiels d'accès à l'emploi selon la période d'installation ou le lieu de naissance sont plus nets.

Chez les hommes, le retour à La Réunion constitue un atout en termes d'accès à l'emploi par rapport aux non migrants pour les niveaux de diplôme intermédiaires (Bac et dans une moindre mesure BEP) et pour les diplômes les plus élevés (DU deuxième et troisième cycle) ${ }^{38}$. Pour les catégories de diplôme inférieur au BEP, l'avantage du retour

37 Âges où les difficultés d'insertion professionnelle sont les plus prégnantes à La Réunion et où les effectifs des natifs de retour après une migration sont les plus nombreux.

38 Pour les niveaux de diplôme les plus élevés : 94,4\% des natifs de retour ont un travail contre $88,1 \%$ des non migrants. 
n'est pas vérifié et ce sont les immigrants nés en métropole qui affichent les taux d'emploi les plus hauts. Les différentiels selon le lieu de naissance sont alors les plus marqués.

Chez les femmes âgées de vingt-cinq à trente-neuf ans, les différences de taux d'emploi entre les différentes populations sont beaucoup plus stables selon le niveau de diplôme.

Les femmes nées en métropole arrivées à La Réunion au cours des années 1990 ont toujours des taux d'emploi inférieurs aux autres catégories de population. À l'autre extrême, les métropolitaines installées dans le département depuis une plus longue date ont les taux d'emploi les plus élevés.

Enfin, les natives de retour de migration occupent moins souvent un emploi que les non migrantes. Ce constat se vérifie pour l'ensemble des niveaux de diplôme, excepté pour les titulaires d'un diplôme de second ou troisième cycle de l'enseignement supérieur. L'écart de taux d'emploi entre les non migrantes et les Réunionnaises de retour est relativement limité, mais stable selon le niveau de diplôme. Ainsi, chez les femmes les plus jeunes ayant terminé leur scolarité, le retour à La Réunion ne constitue pas un avantage en terme d'accès à l'emploi en comparaison de la population non migrante, seules les plus diplômées ont des taux d'emploi supérieurs.

Ainsi à caractéristiques équivalentes, les immigrants nés en métropole et dans une moindre mesure les natifs de retour de migration ont une situation d'emploi plus favorable que celle des natifs non migrants.

\section{Les natifs de retour occupent des emplois plus qualifiés que les non migrants mais nettement moins que les immigrants nés en métropole}

Cet avantage des natifs de retour et plus encore des immigrants nés en métropole sur le marché de l'emploi à La Réunion est confirmé par l'analyse de la qualification des emplois occupés par ces populations.

Les natifs de retour de migration occupent des emplois globalement plus qualifiés que les non migrants, mais ils sont nettement moins représentés que les individus nés en métropole dans les catégories supérieures (cadres et professions intellectuelles supérieures). Plus précisément, $64,6 \%$ des natifs non migrants sont classés dans les catégories " ouvriers » et « employés » contre 57,5\% des natifs de retour de migration et $26 \%$ des individus nés en métropole nouvellement installés dans le département. Chez les femmes, les proportions sont plus hautes : 70,7 \% des non migrantes et seulement $57 \%$ des natives de retour sont « employées » ou « ouvrières », pour 22,7\% des femmes nées en métropole dont l'arrivée est la plus récente.

En outre, ces différentiels se maintiennent largement quand on raisonne à secteur d'activité (public/privé) et à niveau de diplôme équivalent, chez les hommes comme chez les femmes. À titre d'illustration, parmi les hommes salariés du secteur public, seulement $21,2 \%$ des natifs non migrants titulaires d'un diplôme équivalent ou supérieur au bac occupent un poste de «cadre supérieur » contre 51,9\% des hommes nés en métropole 
installés dans l'île avant 1990 et 51,3 \% de ceux arrivés depuis cette date. Les natifs de retour sont sensiblement plus représentés à ce niveau de qualification, mais la différence avec les non migrants est limitée (27,2 \%). Les natifs de La Réunion les plus diplômés accèdent donc proportionnellement moins aux catégories socioprofessionnelles supérieures que les immigrants nés en métropole.

Dans le secteur privé, les titulaires d'un diplôme équivalent ou supérieur au bac salariés sont majoritairement classés en tant que "profession intermédiaire ». Les proportions varient de $42,4 \%$ à 45,2 \% selon le lieu de naissance et la période d'installation des individus. Cependant, à ce niveau de diplôme, les natifs sont proportionnellement plus souvent « employés » et « ouvriers » que « cadres supérieurs » en comparaison des immigrants nés en métropole : 40,8 \% des métropolitains arrivés à La Réunion au cours des années 1990 et 37,1 \% de ceux qui vivaient déjà à La Réunion occupent un emploi de « cadres » contre seulement 11,5\% des natifs restés dans leur département. Les natifs de retour affichent une proportion de « cadres supérieurs » deux fois plus élevée que les non migrants $(23,2 \%)$, ils retirent un avantage de leur expérience de migration, mais ont une situation moins favorable que les immigrants nés en métropole.

Cette différence dans la qualification des emplois occupés selon l'origine, ne concerne pas seulement les plus diplômés. Les immigrants nés en métropole ayant un diplôme inférieur ou égal au BEP occupent beaucoup plus souvent des emplois de " cadres » ou de " professions intermédiaires » que les natifs de l'île dans le secteur public : 8,1\% des métropolitains installés à La Réunion avant 1990 sont dans ce cas contre seulement $1,7 \%$ des non migrants et 3,5\% des natifs de retour. Ces derniers parviennent donc à tirer un bénéfice de leur migration par rapport aux non migrants mais ils restent moins qualifiés que les immigrants nés en métropole. Globalement, les immigrants nés en métropole les moins diplômés du secteur public accèdent plus largement aux emplois à haut niveau de qualification que les natifs de La Réunion. Chez les salariés du privé ayant les niveaux de diplômes les plus bas, les hommes nés en métropole sont nettement moins souvent « ouvriers » et ont des proportions d' « employés » équivalentes aux natifs. La différence avec les natifs s'explique par les proportions de « professions intermédiaires » et de « cadres supérieurs » qui sont plus hautes chez les individus nés en métropole travaillant à La Réunion : $30 \%$ de « professions intermédiaires » et $8 \%$ de « cadres » chez les hommes nés en métropole contre respectivement $14,5 \%$ et $1,7 \%$ pour les natifs de retour, et seulement $9,4 \%$ et $1 \%$ pour les non migrants.

Les différences de qualification des emplois occupés par les salariés du secteur public ou assimilé et du secteur privé selon le lieu de naissance se maintiennent à diplôme égal $^{39}$. Selon le lieu de naissance, ces différentiels se vérifient de la même manière chez

39 L'approche de la qualification des emplois selon le niveau de diplôme détaillé des individus met en avant des différentiels également très nets. Ces écarts ne s'expliquent donc pas par une variation des niveaux de diplôme au sein des catégories regroupées. Par exemple, chez les hommes salariés du secteur public titulaires d'un diplôme de second ou troisième cycle du supérieur : 70,6 \% des métropolitains arrivés à La Réunion entre 1990 et 1999, 69,9\% de ceux installés avant 1990 contre seulement $46,2 \%$ des natifs non migrants et $49 \%$ des natifs de retour occupaient un poste de cadre supérieur. Chez les femmes, les proportions sont respectivement de 53,4\% et 56,8\% chez les « métropolitaines » contre $26,8 \%$ et $38,8 \%$ chez les natives de La Réunion. 
les femmes, elles occupent cependant des emplois globalement moins qualifiés que les hommes. À secteur d'activité et diplôme équivalents, les immigrants nés en métropole semblent être « avantagés » sur le marché du travail de l'île, ils occupent des emplois plus qualifiés que les natifs. Les natifs de La Réunion de retour de migration entre 1990 et 1999 parviennent globalement à tirer un bénéfice de leur expérience de migration. Ils occupent des emplois plus qualifiés que les non migrants et accèdent en plus grand nombre aux catégories supérieures, mais le bénéfice qu'ils tirent de leur expérience de migration est plus réduit que les individus nés en métropole venus travailler dans le département.

\section{CONCLUSION}

À La Réunion, le développement est indissociable de la politique de départementalisation. Le développement de l'île ne peut se comprendre que dans la relation de dépendance entre ce département et sa métropole.

Si du point de vue des indicateurs macro-économiques le développement de l'île peut être considéré comme une réussite, il convient de relativiser ces progrès au niveau individuel en raison du maintien de déséquilibres et d'inégalités marquées qui s'articulent notamment autour de la question de l'emploi.

Dans ce contexte où le développement est inégalement partagé entre les différentes catégories de population, la migration constitue un enjeu essentiel pour une petite société insulaire comme La Réunion. Elle a presque toujours été présentée comme la solution adéquate pour freiner la croissance démographique et résoudre les problèmes économiques et sociaux qui en découlent. Elle constitue encore aujourd'hui un outil d'ajustement essentiel pour répondre aux déséquilibres du marché du travail (surplus de main-d'œuvre pas ou peu qualifiée, pénurie de main-d'œuvre qualifiée). Cependant, alors que les sorties du département étaient plus nombreuses que les entrées depuis la départementalisation, les dernières décennies du XXe siècle ont été marquées par une augmentation des arrivées et des retours entraînant un effet positif de la migration sur la croissance démographique. L'élévation du nombre de personnes en âge de travailler accroît la concurrence entre les individus ce qui crée des tensions sur un marché du travail au dynamisme insuffisant. Dans ce contexte, toutes les populations ne tirent pas les mêmes avantages de la migration. On l'a vu, l'émigration des natifs en métropole leur permet d'accéder plus largement à l'emploi, ce qui devrait encourager au départ. Cependant, les migrants les plus récemment installés rencontrent plus de difficultés d'insertion. En outre, si les natifs de la Réunion en métropole accèdent plus largement à l'emploi que les non migrants c'est en partie parce qu'ils occupent en plus grande proportion des emplois à niveau de qualification plus faible. La diminution des opportunités d'emploi en métropole pour les migrants les plus récents pourrait expliquer une moindre inclination au départ et inversement soutenir les flux de retour. Néanmoins, leur départ est globalement profitable d'un point de vue individuel, car il leur permet d'élever leur niveau de formation et d'acquérir une expérience professionnelle hors du département, ce qui renforce leurs capacités et augmente leurs possibilités d'insertion sur le marché du travail en cas de retour. Les natifs de retour dans leur département après avoir migré et surtout les individus nés en métropole venus s'installer à La Réunion ont un niveau d'étude élevé qui leur permet de 
bien s'insérer sur le marché du travail de l'île demandant de plus en plus de qualifications. À l'inverse, les natifs non migrants ayant un niveau de diplôme plus bas, connaissent une insertion difficile, ils accèdent moins souvent à l'emploi et quand ils y parviennent, ils occupent des emplois moins qualifiés.

À secteur d'activité et diplôme équivalents, on note des différentiels sensibles en termes de qualification des emplois occupés selon l'origine, le fait d'avoir connu une migration ou encore l'âge et le sexe des individus. L'analyse de ces différentiels, entre préférences et désavantages, doit être approfondie à partir d'enquêtes spécifiques mesurant plus précisément les parcours scolaires, professionnels ou encore migratoires des individus ${ }^{40}$ qui seules permettraient d'estimer plus précisément les sources des bénéfices ou désavantages (expérience professionnelle, capital scolaire, qualifications, etc.). Il apparaît ainsi que la concurrence entre les individus est particulièrement forte à La Réunion sur un marché de l'emploi saturé et sélectif. Aussi, si la migration constitue un outil essentiel dans le développement des économies insulaires, elle peut également contribuer à accroître les inégalités entre migrants et non migrants et être source de tensions sociales. A l'heure où le niveau d'éducation de la population native progresse, le recours à l'immigration de main-d'œuvre extérieure et au maintien d'avantages à l'installation peuvent paraître moins justifiés. Les débats sur les thèmes de la "préférence régionale » ou de la « sur-rémunération » des fonctionnaires qui ont largement été relayés par la presse locale illustrent la place centrale qu'occupent ces questions de migrations, mobilités et d'emploi dans l'opinion publique. Dans l'autre sens, depuis le début de la décennie 2000, la migration/mobilité des jeunes natifs est présentée par les autorités publiques comme un instrument au service du développement et de l'épanouissement individuel. Les pouvoirs publics misent sur la formation et l'enseignement supérieur, que ce soit localement à l'université de La Réunion ou via les programmes de mobilité qui aident les jeunes à suivre des études en métropole ou à l'étranger. L'orientation de la politique de mobilité actuelle apporte une nouveauté par rapport aux grands programmes des décennies 1960 et 1970, puisqu'elle se place dans la perspective d'un retour profitable et non pas d'une émigration de longue durée, voire définitive. Ces programmes sont récents et il est encore trop tôt pour en mesurer l'ensemble des effets, tant sur le volume et la nature des flux que sur le développement local et notamment la structure des diplômes de la population résidant à La Réunion.

\section{Références bibliographiques}

ACADÉMIE de La Réunion (2004) Repères Statistiques 2003-2004, Réunion, Service statistique académique, $110 \mathrm{p}$.

AFD-INSEE-IEDOM (2005) Émergence de nouveaux moteurs de croissance. Le rattrapage économique réunionnais expliqué, 1993-2002, Réunion, INSEE, 48 p.

AFD-INSEE-IEDOM (2004) Une double transition presque réussie. Chômage, productivité et politique d'emploi à La Réunion, Réunion, INSEE, $56 \mathrm{p}$.

40 À ce titre, l'enquête Ined-Insee « Migrations, famille et vieillissement » dans les Dom, dont les données sont attendues courant 2011, devrait apporter des éléments permettant d'approfondir ces premières analyses menées à partir des recensements de la population.

REMI 2011 (27) 3 pp. 131-164 
BRETON Didier et TEMPORAL Franck (2010) Des enfants de plus en plus tard ? Territoires de résistances en outremer, Chaire Quételet 2010, retournements et résistance de la fécondité dans les pays $d u$ Sud, Louvain, $20 \mathrm{p}$.

CATTEAU Christine (2003) Des maladies de civilisation, Économie de La Réunion, 116, pp. 23-25.

CHANE-KUNE Sonia (1996) La Réunion n'est plus une île, Paris, L'Harmattan, 368 p.

CHEUNG CHIN TUN Yvon (2000) L'enseignement secondaire, Économie de La Réunion, 105, pp. 12-16.

CHEVALIER François (2001) Migrations : la nouvelle vague reste d'une ampleur modérée, Économie de La Réunion, 107, pp. 24-26.

DOMENACH Hervé et PICOUET Michel (1992) La dimension migratoire des Antilles, Paris, Economica, $254 \mathrm{p}$.

FESTY Patrick et HAMON Christine (1983), Croissance et révolution démographiques à La Réunion, Paris, PUF, 116 p.

GUILMOTO Christophe et SANDRON Frédéric (2003) Migration et développement, Paris, La documentation française, $142 \mathrm{p}$.

INSEE (1987) Spécial migration, Économie de La Réunion, 29-31.

LOPEZ Albert (1996) Une révolution sanitaire déclenche la transition démographique, Économie de La Réunion, 82-83, pp. 6-12.

MARIE Claude-Valentin et TEMPORAL Franck (2001) Migrants de retour : une émigration très encadrée par les institutions, Économie de La Réunion, 109, pp. 12-14.

PARAIN Claude (1999) Le déclassement des diplômés, Économie de la Réunion, 102, pp. 20-22.

PARAIN Claude (1996) L'emploi se transforme et le chômage apparaît, Économie de La Réunion, 82-83, pp. 27-31.

PAYET Fabrice (2000) Le Bac confirme l'échelle sociale, Économie de La Réunion, 105, pp. 16-18.

RALLU Jean-Louis (1997a) La population des DOM. Évolution récente, migration et activité, Population, 52 (3), pp. 699-727.

RALLU Jean-Louis (1997b) Population, migration et développement dans le Pacifique Sud, Paris, Unesco-Orstom-Ined, $224 \mathrm{p}$.

RALLU Jean-Louis et MARIE Claude-Valentin (2004) Migrations croisées entre DOM et métropole : l'emploi comme moteur de la migration, Espaces, Populations et sociétés, 2, pp. 237-252.

TEMPORAL Franck (2006) Synthèse des études existantes sur la pauvreté à La Réunion, in Les travaux de l'Observatoire National de la Pauvreté et de l'Exclusion Sociale 2005-2006, Paris, La Documentation française, pp. 465-510

WIDMER Isabelle (2005) La Réunion et Maurice. Parcours de deux îles australes des origines au XXe siècle, Paris, INED, 291 p. 


\title{
Migrations et emplois à l'île de La Réunion
}

\author{
Franck Temporal
}

Depuis sa départementalisation en 1946, l'île de La Réunion a connu de profondes mutations et des transitions multiples (politique, épidémiologique, démographique, familiale, économique et sociale) dont la caractéristique commune est leur simultanéité et leur rapidité. Aujourd'hui, le niveau de développement de La Réunion est élevé, mais il convient de relativiser le caractère positif de ces évolutions au niveau individuel en raison du niveau élevé du chômage et du maintien d'inégalités sociales marquées. Cette recherche s'interroge sur les relations entre migrations et développement à La Réunion en s'appuyant sur une analyse quantitative des données des recensements de la population. L'analyse porte notamment sur les effets démographiques et les conséquences sociales des migrations.

\section{Migration and Employment on Reunion Island}

\section{Franck Temporal}

Since 1946 and the integration of the former colony of La Réunion into the national territory (département) this island territory has gone through profound change and numerous transitions (political, epidemiological, demographic, family, economic and social) whose common feature is their simultaneity and their speed. Today, the level of development of Reunion Island is high, but the positive nature of this transition at the aggregate level needs to be set against individual level trends, notably high unemployment and persisting social inequalities. This study examines the relationship between migration and development in Reunion Island based on analysis of population census data. In particular, we examine the demographic and social consequences of migration.

\section{Migración y el empleo en la isla de Reunión}

\author{
Franck Temporal
}

Desde la departamentalización en 1946, la isla de Reunión ha experimentado profundos cambios y transiciones múltiples (políticos, epidemiológicos, demográficos, familiares, económicos y sociales) cuya característica común es su simultaneidad y rapidez. Hoy en día, el nivel de desarrollo de La Reunión es alto, pero debe relativizarse el punto de vista positivo de estos avances a nivel individual a causa del alto nivel de desempleo y de las desigualdades sociales existentes. Esta investigación examina la relación entre migración y desarrollo en La Reunión basandose en un análisis cuantitativo de los censos de población. En particular se analysa los efectos demográficos y consecuencias sociales de la migración. 